\title{
Tactical Waste Collection: Column Generation and Mixed Integer Programming Based Heuristics
}

\author{
Jens Van Engeland*1a, Jeroen Beliën ${ }^{\mathrm{b}, \mathrm{c}}$ \\ ${ }^{a}$ KU Leuven, Center for Economics and Corporate Sustainability, Faculty of Economics and Business, Campus Brussels \\ (Belgium) \\ ${ }^{b}$ KU Leuven, Center for Information Management, Modeling and Simulation, Faculty of Economics and Business, Campus \\ Brussels (Belgium) \\ ${ }^{c}$ KU Leuven, Research Centre for Operations Management, Faculty of Economics and Business, Campus Leuven (Belgium)
}

\begin{abstract}
Environmental considerations and corresponding legislation cause a shift from waste management to materials management, requiring efficient collection of these flows. This paper develops a model for building tactical waste collection schemes in which a set of capacitated vehicles visits a set of customers during a given time period. Each vehicle must visit the disposal facility to discharge the waste after each customer visit. This is motivated by the fact that the waste of each customer has to be weighed at the disposal facility. The goal is to find a set of routes for each vehicle that satisfy both the demand and the frequency constraints and minimize the total cost. Since a state-of-the-art solver could not find a solution with a reasonable gap within an acceptable time limit, a column generation and a mixed integer programming based heuristic are proposed. While the mixed integer programming based heuristic outperforms the column generation heuristic in terms of solution quality, the lower bound provided by column generation allows to prove the small optimality gaps of the solutions obtained. Moreover, by applying both heuristics on instances derived from real-life data, they proved to be capable of finding good quality solutions in small computation times.
\end{abstract}

Keywords: OR in service industries, waste collection, mixed integer programming, column generation, tactical level planning

\footnotetext{
*corresponding author. Address: Warmoesberg 26, BE-1000 Brussels

Email addresses: jensve@gmail.com (Jens Van Engeland*1), jeroen.belien@kuleuven.be (Jeroen Beliën)

${ }^{1}$ supported by a Ph. D. fellowship of the Research Foundation - Flanders (FWO)
} 


\section{Introduction}

Due to environmental awareness and corresponding legislation (see, e.g., the EU circular economy strategy (European Commission, 2017), attention is shifting from waste management to materials management. The "EU revised legislative proposals on waste" set ambitious goals: a common EU target of $65 \%$ and $75 \%$ for recycling municipal and packaging waste respectively by 2030. The focus on separate waste and material flows will have an undeniable impact on efficient collection schemes.

This research was inspired by a real-life problem in the context of collecting municipal solid waste, and hence a customer can be seen as a neighbourhood (sequence of streets), or even an entire municipal district, borough, municipality or suburb. The model is generic, though, and is also applicable to other contexts; e.g., collection at companies or waste recycling centres which have several containers installed and where collection time thus depends on the amount of waste. Strategic decisions like the location of vehicle depot and disposal facility are assumed to be fixed. Also operational decisions like vehicle routing inside a neighbourhood (customer) fall outside the scope of this paper.

The focus of this paper is thus on the design of a tactical collection scheme. The goal of a tactical collection scheme is to assign to each vehicle for each day a set of customers to be visited. The objective is to minimize the total cost of this allocation. A unique constraint is that each vehicle must visit the disposal facility after each customer visit before continuing collection at other customers. The reason for this is that, due to payment issues, the collected waste of each customer has to be weighed separately at the disposal facility (and weighing is not possible during collection as vehicles do not have a weighing device).

Once the tactical schedule has been developed, each municipality can be divided in as many neighborhoods (zones) as there are collection days in the schedule for the given municipality. An example is given in the operational collection scheme in Figure1. Each zone (green and blue) corresponds to a group of streets such that the (estimated) total waste collected in these streets is equal to the waste that needs to be collected at the given municipality on that specific day in the tactical schedule. In order to ensure that each municipality can deal with this grouping problem, and to avoid that the number of collection days for a municipality is impractically large, the number of zones needs to be kept sufficiently small. Likewise, in the context of waste collection at companies or waste recycling centres it is reasonable to assume that collection is performed on a limited number of days in the planning horizon. To this aim, we impose an upper bound on the number of visits to a municipality in the given planning horizon.

In conclusion, the problem studied in this paper differs from a traditional vehicle routing problem (VRP)

in (a) the focus on tactical decision-making excluding the routing decision, and (b) the upper limit on the number of visits to each customer, whereas traditional service level constraints normally impose a lower limit on the number of visits per customer.

The main contributions of this paper are as follows:

- we model a real-life tactical waste collection problem;

- we develop a basic mixed integer programming (MIP) model;

- we propose two different heuristic approaches: a column generation (CG) approach and a MIP-based (2 trip) heuristic;

- based on the CG approach, we propose two different ways of calculating strong lower bounds. 


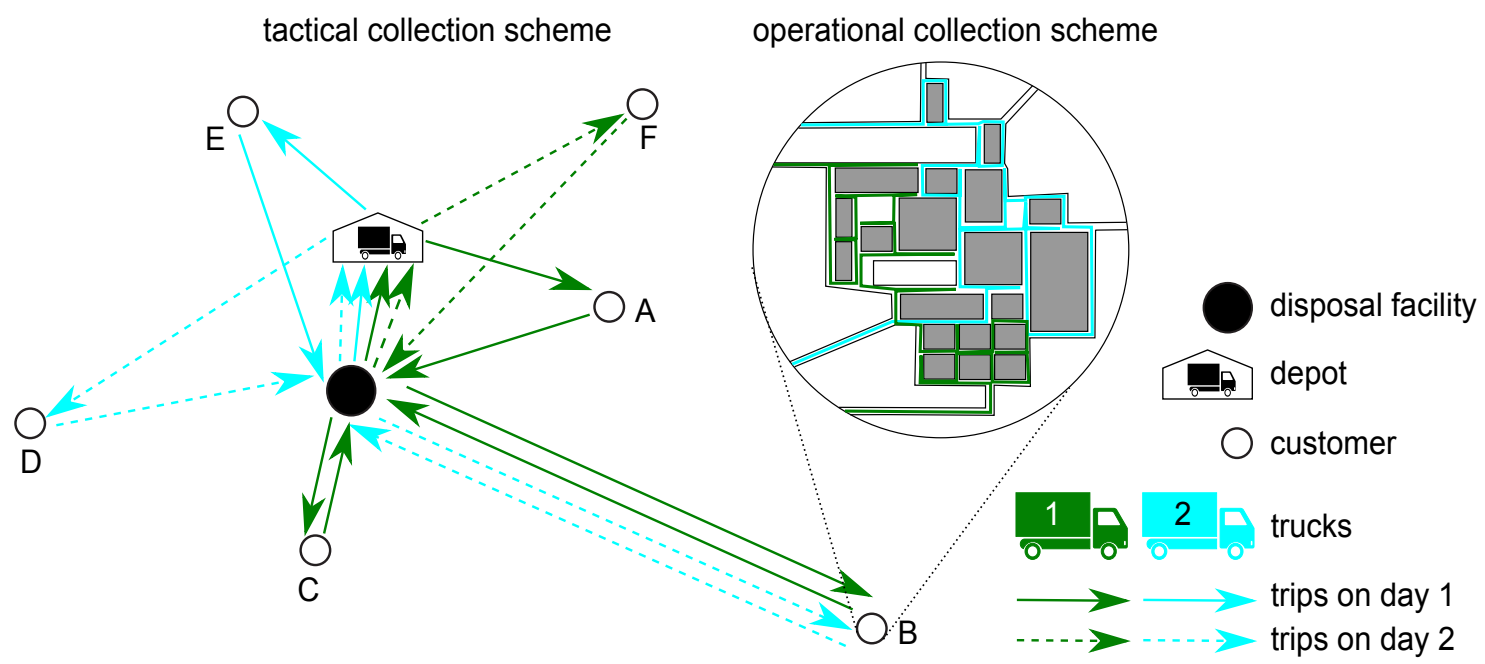

Figure 1: Example of a tactical and an operational collection scheme. Design of the latter will not be considered in this paper.

The remainder of this text is organized as follows. Section 2 discusses related literature, while Section 3 states the problem. Section 4 presents the column generation and MIP-based heuristic solution approaches, which is followed by a computational experiment and analysis of results in Section 5 . Finally, Section 6 concludes this paper.

\section{Literature review}

Beliën et al. (2014) classify the literature on waste collection problems into different categories such as the scope, objective, constraints and solution method. One conclusion is that heuristic approaches are more common than exact approaches. Ghiani et al. (2014) provide an overview of the literature on strategic and tactical issues in operations research models applied to solid waste management. The strategic decisions comprise the choices on type, number and location of facilities (Mansini \& Speranza, 1998) and global waste flows towards them (Ghiani et al., 2014), while the tactical decisions concern vehicle fleet design, crew decisions and solid waste collection zone definition (also known as sectoring or districting).

The literature on tactical models appears to be scarce. le Blanc et al. (2006) address tactical decisions related to end-of-life product flows, while Gomes Salema et al. (2009) study closed loop supply chain design. The latter develop a location-allocation model for forward and reverse flows over a planning horizon. However, their focus is on a strategic-tactical level instead of the tactical level considered in our paper. Ignall et al. (1972) model the assignment of crews to shifts and days in a way that costs and uncollected waste are balanced. A planning horizon for a single district is considered. The contribution of Mansini \& Speranza (1998) is to evaluate separate waste collection at a tactical level. The decision variables are the amounts of waste to collect on each day of the planning horizon. Their objective is to minimize the peak of waste, i.e., the maximum amount that has to be collected on a given day. List et al. (2006) focus on fleet sizing and equipment acquisition for disposition of radioactive wastes. In a two-phase robust optimization model, they decide on the fleet size and equipment acquisition (first stage) and eventual waste flows and truck use (second stage). Ghiani et al. (2013) consider a tactical planning problem in the waste management sector in which vehicles and staff have to be allocated to tasks over a given planning horizon. Bish (2011) 
proposes a model designed for bus-based evacuation. The problem differs substantially from a traditional VRP, and resembles planning at a tactical level. A heuristic algorithm is developed to solve the problem. Also Hernandez et al. (2017) study a tactical problem, of which the solutions could be used as a blueprint for a posteriori operational planning. In their paper, a variant to the periodic vehicle routing problem (PVRP) is solved using two heuristics.

While these papers focus solely on the tactical level, other studies have integrated tactical with operational decision levels. Angelelli \& Speranza (2002) model three waste-collection systems to estimate operation costs. A solution of their model consists of a set of routes, assigned to a vehicle on a certain day of the planning horizon. Every collection point has to be visited according to a feasible collection scheme and at most one time per day. le Blanc et al. (2006) optimize the collection of end-of-life vehicles using a VRP in which only two customers can be visited in one tour. The problem is solved using a two-step heuristic. Andrea Arribas et al. (2010) propose an efficient design for solid waste collection in which the fleet composition and districting decisions are tackled using linear integer programming models and the routing decisions are handled using a tabu search heuristic. Cortinhal et al. (2016) present a hill climbing and a tabu search heuristic to simultaneously solve the districting problem, with the objective of balanced and connected sectors, and the trip construction problem, with the objective of minimizing total traveling time. Similar objectives of balancing workload at depots and minimizing the route distances are considered by Ramos \& Oliveira (2011). Motivated by a real-life commercial waste collection problem, Kim et al. (2006) incorporate time windows in their problem setting. They propose an extended insertion algorithm and a clustering-based algorithm for tackling the districting problem, after which a single vehicle trip is built for each cluster. The districting problem's objective is to maximize workload balance between the routes and proximity among the stops of the same route, while the vehicle routing minimizes total traveling time. Constantino et al. (2015) propose an exact method, based on a model that simultaneously designs sectors and builds routes, and a heuristic solution method which sequentially solves the two problems in a real-world waste collection context. Their districting objective is to minimize overlapping of vehicle routes, measured in terms of the number of nodes that are common to the tasks of different routes. The resulting districts exhibit 'nice' characteristics, i.e. maximal connectivity and compactness, for implementation in practice. Mourão et al. (2009) develop two two-phase heuristics and one best insertion heuristic to integrate district definition and trip construction in urban waste collection networks. The heuristics promote workload balance, compactness, and contiguity. Similar to our problem, sectors are limited in workload time and the objective of the trip construction phase is to minimize total duration of trips. The authors conclude that none of the heuristics is dominant if both traveling distance and sector quality measures are considered.

The operational level concerns routing and scheduling problems (Mansini \& Speranza, 1998). The related literature on waste collection problems addresses many variants of the PVRP. In the PVRP with Balance Constraints (PVRP-BC) one wants to maintain a balanced workload among the drivers (Jang et al., 2006). A vehicle fleet based across a number of depots leads to the formulation of the Multi-Depot PVRP (MDPVRP) (Baldacci et al. 2011), where periodic deliveries are made using this fleet. Another variant is the PVRP with Service Choice (PVRP-SC), introduced by Francis et al. (2006), in which the visit frequency is considered as variable within the model. This decision is mostly subject to a hard constraint that ensures an exact or a minimal number of visits. A recent overview on the research of PVRP and more of its variants can be found in the review by Campbell \& Wilson (2014), or in the work by Toth et al. (2014). 
Our study differs from these integrated or operational approaches in the fact that our collection trips do not explicitly involve node or arc sequencing due to the constraint that after every node visit the collected waste needs to be disposed at the drop-off location. Secondly, in contrast to studies mentioned above, we do not assume that one district will be handled by one vehicle on a single day. Contrarily, our problem involves the construction of a cyclic timetable that schedules the node visits subject to a maximum number of visits per node constraint. In the context of municipal waste collection, the output of our approach, i.e. the number of vehicles required, the scheduled visits to each municipality and the amounts to be collected during each visit, will determine the input for the districting problem in which streets are grouped into districts such that the amount of waste to be collected matches the amount prescribed in the collection schedule obtained. Our problem thus focuses on the tactical level determining a fleet composition and a cyclic collection timetable taking into account the required time and vehicle capacities for the collection task.

\section{Problem statement}

The problem consists of the design of a tactical collection scheme for a certain type of municipal solid waste. Such a collection scheme is a cyclic schedule that prescribes the amount of waste to collect per day at each municipality. The model developed in this paper is only applicable to materials that can be let for collection for a certain period (number of days) which is the case for paper and carton, and plastic, metal and drink cartons (PMD), but for instance not for dangerous waste that needs to be collected the same day as it is generated. Additionally, our model only holds for settings in which it must be allowed to pick-up only part of the waste of a given municipality at a certain day. Note that this does not mean that for a given municipality a part of the waste, that is put outside for collection, is not collected the same day. In our setting, each household will only have one fixed, periodic day on which waste is collected. To clarify this: if the tactical collection scheme prescribes that, for instance, $30 \%$ of the total waste of municipality $\mathrm{x}$, is collected on Tuesday and $70 \%$ on Thursday, it means that a total number of streets representing $30 \%$ of municipality x's waste is collected on Tuesday, while the remaining $70 \%$ of the streets (households) are served on Thursday. Note that each household in municipality $\mathrm{x}$ will have only one fixed day (= periodic pick-up day) in the cycle (in which the cycle is one or two weeks). Starting from a tactical schedule, operational schedules could be built (see Figure 1). An operational waste collection scheme defines, within a customer area, the vehicle routes in much more detail (e.g., on street level).

Efficient tactical collection schemes can substantially decrease the costs, which comprise vehicle costs and collection costs (or route costs). The vehicle costs can be minimized by using a minimal number of vehicles. The collection costs are driven by the durations of the different vehicle routes, which impact the fuel costs and person-hour costs, respectively.

The following constraints are considered:

1. vehicle capacity restrictions: the amount of waste collected could never exceed a vehicle's capacity. If capacity is reached, a trip to the disposal facility should be performed.

2. an upper bound on the number of working hours: since a daily schedule of a vehicle should be performed by a single crew, there is a limit on the number of working hours. The working hours depend on the travel times and on the amounts of waste collected at the customers.

3. an upper bound on the maximal number of different collection days: during the planning horizon, there is a maximum limit on the number of days on which collection can take place at each customer. The reason for this is explained in Section 1 . 
4. covering restrictions: the demand constraints state that the customer's demand should be satisfied, i.e., all waste of each customer should be collected in the given cycle period.

Input data consist of a set of customers, each with a location and a given demand (waste to be collected), a depot location and a disposal facility location. Contrary to an operational collection scheme, vehicles cannot travel between customers (see Section 1). However, a vehicle can make several trips per day and a distinction is made between type 1 and type 2 trips. In a type 1 trip the vehicle starts at the depot to visit a customer. When the waste is collected, the vehicle drives to the disposal facility. In a type 2 trip, a vehicle starts at the disposal facility, visits a customer to collect waste, and then drives back to the disposal facility. The reason for this distinction between type 1 and type 2 trips is that they have a different travel time and hence a different cost. The costs for both trips are equal to a fixed cost per hour (which covers both expenses for fuel and personnel) multiplied by the travel time of the respective trip. At the end of the day, the vehicle travels from the disposal facility back to the depot. The required travel time of this end of the day trip is added to the traveling duration (and hence cost) of a type 1 trip. Note that this is possible because we assume only one disposal facility and one depot. Hence, each type 1 trip automatically leads to a journey from the disposal facility to the vehicle depot at the end of the day (directly or after 1 or more type 2 trips). When combining one type 1 trip with a number of type 2 trips, one can construct a route. A route is a combination of trips and hence prescribes which customers a vehicle has to visit on a particular day.

Figure 1 depicts an example of two vehicles each performing two routes (one per day). The first (green) vehicle starts the first day with a visit to customer A and delivers at the disposal facility which is (the first part of) a type 1 trip. Afterwards, it collects at customer B before returning to the disposal facility (type 2 trip). Finally, it visits customer C (type 2 trip) before it returns to the depot (final part of type 1 trip). On the first day the second (blue) vehicle only performs a type 1 trip to customer E, unloads at the disposal facility and returns to the vehicle depot. On the second day the green vehicle only performs a type 1 trip to customer F, whereas the blue vehicle visits $\mathrm{D}$ with a type 1 trip and $\mathrm{B}$ with a type 2 trip.

We assume identical vehicles and days as well as symmetric travel times of the type 2 trips, i.e., back and forth are assumed identical. Although workload balancing among the different vehicles is not incorporated, it is still possible to strive for balanced workloads when assigning crews to vehicles at a later stage.

The problem under study can be formulated as a MIP, with sets and indices:

$$
\begin{array}{ll}
m \in M & \text { customers } \\
d \in D & \text { days in the planning horizon } \\
v \in V & \text { vehicles }
\end{array}
$$

The parameters are [units of measurement]: 


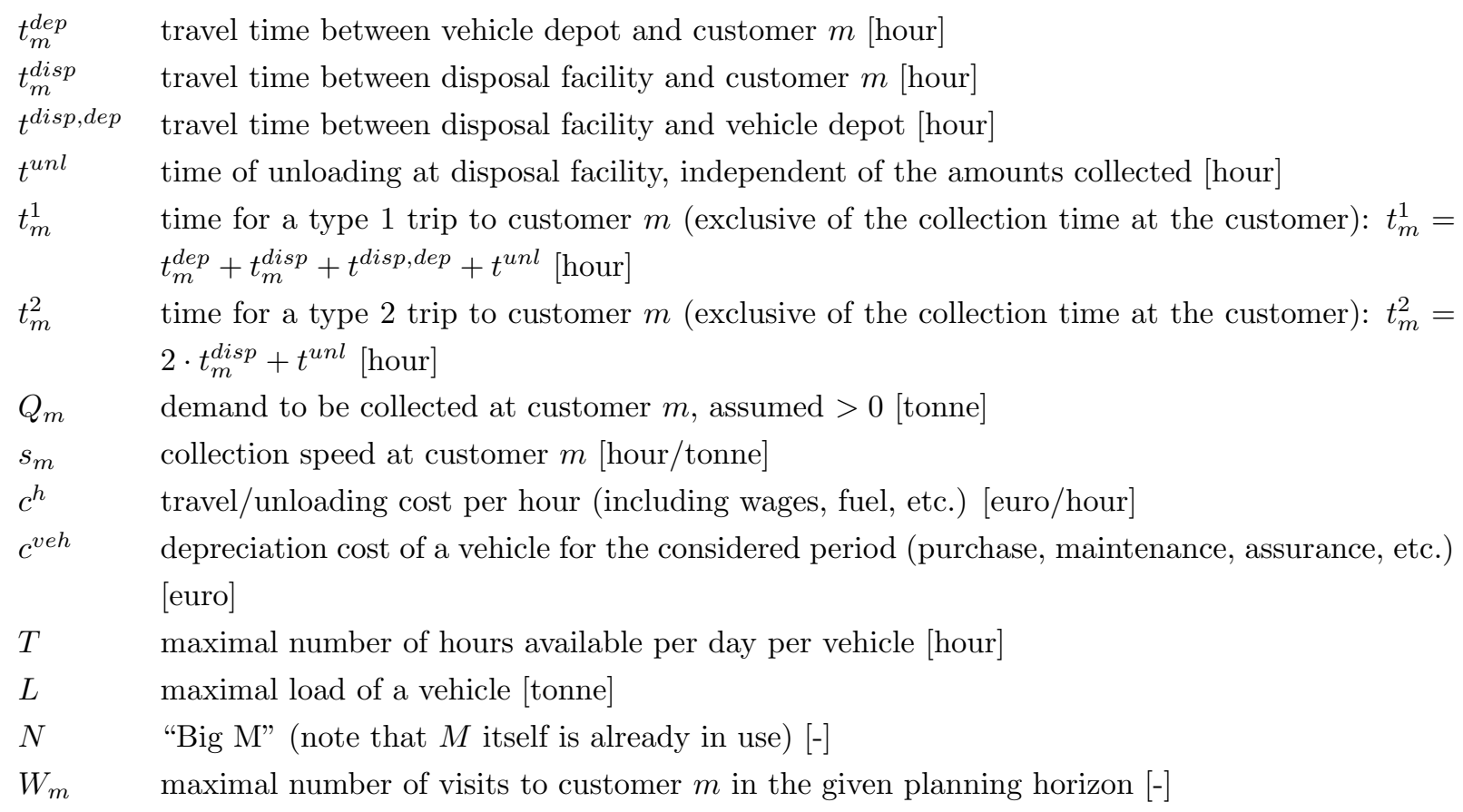

The decision variables are:

$y_{v m d}^{1}=1$ if vehicle $v$ is used to serve customer $m$ on day $d$ on a type 1 trip, 0 otherwise

$y_{v m d}^{2} \quad$ number of type 2 trips by vehicle $v$ to customer $m$ on day $d$

$x_{v m d}^{1} \quad$ total load collected by vehicle $v$ at customer $m$ on day $d$ on type 1 trip [tonne]

$x_{v m d}^{2} \quad$ total load collected by vehicle $v$ at customer $m$ on day $d$ on all type 2 trips [tonne]

$z \quad=$ total number of vehicles needed

$w_{m d}=1$ if customer $m$ is visited on day $d, 0$ otherwise

The tactical waste collection problem (TWCP) can be formulated as follows:

$$
\text { minimize } \quad c^{v e h} z+\sum_{v \in V} \sum_{m \in M} \sum_{d \in D}\left(c^{h}\left(t_{m}^{1} y_{v m d}^{1}+t_{m}^{2} y_{v m d}^{2}\right)\right)
$$


subject to

$$
\begin{aligned}
& x_{v m d}^{1} \leq L \cdot y_{v m d}^{1} \quad v \in V, m \in M, d \in D \\
& x_{v m d}^{2} \leq L \cdot y_{v m d}^{2} \quad v \in V, m \in M, d \in D \\
& \sum_{v \in V} \sum_{d \in D}\left(x_{v m d}^{1}+x_{v m d}^{2}\right)=Q_{m} \\
& \sum_{m \in M}\left(s_{m}\left(x_{v m d}^{1}+x_{v m d}^{2}\right)+t_{m}^{1} y_{v m d}^{1}+t_{m}^{2} y_{v m d}^{2}\right) \leq T \\
& \sum_{m \in M} y_{v m d}^{1} \leq 1 \\
& N \sum_{m^{\prime} \in M} y_{v m^{\prime} d}^{1} \geq y_{v m d}^{2} \\
& z \geq \sum_{v \in V} \sum_{m \in M} y_{v m d}^{1} \\
& w_{m d} \geq y_{v m d}^{1} \\
& N w_{m d} \geq y_{v m d}^{2} \\
& \sum_{d \in D} w_{m d} \leq W_{m} \\
& y_{v m d}^{1} \in\{0,1\} \\
& y_{v m d}^{2} \in\{0,1,2, \ldots\} \\
& x_{v m d}^{1}, x_{v m d}^{2} \geq 0 \\
& w_{m d} \in\{0,1\} \\
& m \in M \\
& v \in V, d \in D \\
& v \in V, d \in D \\
& v \in V, m \in M, d \in D \\
& d \in D \\
& v \in V, m \in M, d \in D \\
& v \in V, m \in M, d \in D \\
& m \in M \\
& v \in V, m \in M, d \in D \\
& v \in V, m \in M, d \in D \\
& v \in V, m \in M, d \in D \\
& z \in\{0,1,2, \ldots\}
\end{aligned}
$$

Objective function (1) minimizes the sum of the vehicle depreciation costs and the total route costs, incurred by performing type 1 and type 2 trips. Constraints $(2)$ and constraints $(3)$ model the vehicle capacity restrictions during type 1 and type 2 trips, respectively. Constraints (4) make sure that the demand of each customer is met. Constraints (5) restrict the number of working hours per vehicle per day. Constraint set (6) ensures that each vehicle can perform at most one type 1 trip per day. Constraint set (7) makes sure that type 2 trips of vehicle $v$ on day $d$ can only be performed if there is a type 1 trip for vehicle $v$ on day $d$. This constraint implies that every vehicle must start and end at the depot. Constraint (8) calculates $z$ to equal the maximal number of type 1 trips per day. This is the number of vehicles that will be required. Constraint sets (9)-10) ensure that if a vehicle visits customer $m$ on day $d$ the corresponding $w_{m d}$ variable is forced to have value 1. Subsequently, constraints (11) together with constraints (9)-(10) ensure that a customer can be visited at most $W_{m}$ times during the planning horizon. Finally, constraints (12)-(16) define the domains of the decision variables. To guarantee that a feasible solution will be found, the number of available vehicles $|V|$ should be large enough. We make $|V|$ equal to the minimal number of vehicles needed if all collection would be performed using only type 1 trips. This fleet size is found by a small IP given in Appendix A.

Proposition 1. TWCP is strongly NP-hard.

Proof: Our proof is based on reduction from the bin packing decision problem, which is known to be strongly NP-complete. Consider an instance in which all vehicles are identical and with the following parameters: a 
one-day planning horizon (index $d$ is dropped), the capacity of a vehicle is infinite, $c^{v e h}=1$ and all other costs are equal to zero. All vehicles have the same properties (homogeneous fleet) and the maximal time available for each vehicle on the considered day equals $T$. Given a planning horizon of one day, the constraint on the maximally allowed number of visits per customer has become redundant. Furthermore, we assume the vehicle depot and disposal facility are on the same location, hence the travelling times for type 1 and type 2 trips are equal and have values between 0 and $T$ : $0<t_{m}^{1}=t_{m}^{2}<T$ for all $m$. Moreover, the sum of all travelling times must equal $\sum_{m} t_{m}^{1}=\sum_{m} t_{m}^{2}=Z \cdot T$ in which $Z$ is an integer $>1$. The required collection times at the customers to collect all the waste are zero, i.e., $s_{m}=0$ for all $m$. In the TWCP decision problem we need to determine whether there exists a feasible allocation of customers to vehicles such that all the waste is collected and the total cost is at most $Z$, i.e., the number of vehicles used is $Z$. Therefore, the customers need to be divided into $Z$ subsets. Each such subset consists of customers that will be visited by a certain vehicle such that the total travel time of the vehicle does not exceed $T$. This is equivalent to the bin packing decision problem in which each bin has capacity $T$. It can be verified that the minimum number of bins equals $Z$ if and only if the minimal cost of this TWCP equals $Z$. Hence, bin packing is a special case of TWCP and TWCP is strongly NP-hard.

\section{Solution approach}

Since we consider identical vehicles and days, (F1) can be extended by symmetry breaking constraints (SBCs) to strengthen the Linear Programming (LP) relaxation. We experimented with adding various classes of SBCs to reduce the symmetry in vehicle-customer combinations and in customer-day allocations. We also added valid inequalities that enforce that a type 1 trip is performed to a customer that is relatively closer to the depot than to the disposal facility, as compared to other customers visited by vehicle $v$ on day $d$ on type 2 trips. Unfortunately, applying MIP model (F1) both with and without symmetry breaking constraints and valid inequalities on two realistic problem instances using state-of-the-art software (IBM ILOG CPLEX Optimization Studio, Version 12.6) could not solve the problems to optimality or even did not result into a solution with an acceptable gap within a reasonable time limit. For more details on these SBCs and other valid inequalities, including computational results, we refer to Van Engeland (2019). In what follows, we develop a decomposition approach solved by column generation (Section 4.1) as well as a MIP-based heuristic (Section 4.4 which are successful in finding high-quality solutions within reasonably small computational efforts.

\subsection{Heuristic column generation approach}

Column generation (CG) is an iterative approach to solve linear programs for which the number of variables is so large that it is intractable to consider them all explicitly. Based on the premise that only a small number of variables will have a positive value in an optimal solution anyway, only a small subset of variables is considered explicitly. To apply CG, the original problem is split into two problems: a master problem and

a subproblem. The master problem is a reformulation of the original problem in which some constraints are omitted and the original variables are aggregated in subsets that form new decision variables (or columns). Through these columns the omitted constraints are implicitly satisfied. After solving the restricted master LP, the optimal dual values can be used to find one or more new columns to be added to the master LP. The latter is done through solving a subproblem, called the pricing problem, of which the objective is to find a new column with (most) negative reduced cost (if the master LP's objective is minimization). DantzigWolfe decomposition is a popular technique to decompose the original problem into a master-subproblem 
decomposition. For more information on CG and Dantzig-Wolfe decomposition we refer to Desaulniers et al. (2005) and Lübbecke (2011). CG is a popular solution method for the VRP and its variants (Costa et al. 2019). For instance, a vehicle and crew scheduling problem is solved by Bartodziej et al. (2009) using a CG approach and a local search meta-heuristic.

In formulation (F1), most of the constraints are formulated for vehicle-day combinations. With DantzigWolfe decomposition on the variables with indices $v$ and $d$, these constraints can already be satisfied outside the master MIP, when generating the columns. Each column thus represents a feasible one-day schedule for a vehicle, which will be called a 'route' and consists of exactly one type 1 and a certain number of type 2 trips. These columns satisfy constraints (2)-(3), (5)-(7) and (9)-(10). The demand constraints (4), the constraints determining the number of vehicles needed (8) and the constraints limiting the number of times a customer is visited (11) remain in the master problem. Since all vehicles are identical, it suffices to generate columns for a day regardless of the vehicle. Hence, a route defines a combination of customers for a particular day. The following extra notation is needed for the column generation approach:

$k \in K, \hat{K} \quad$ routes (columns). Note that $\hat{K}$ denotes the restricted column set.

This set contains all start columns (see Section 4.2 and all routes generated so far.

It is a subset of the set of all possible routes: $\hat{K} \subset K$.

$p_{k} \quad$ total cost for route $k$

$a_{k m} \quad$ total load (tonnes) collected at customer $m$ in route $k$

$g_{k m d} \quad=1$ if customer $m$ is visited on day $d$ in route $k ; 0$ otherwise

$h_{k d} \quad=1$ if route $k$ is a day $d$ route; 0 otherwise

$r_{v k} \quad$ variable indicating if route $k$ is selected for a vehicle $v$

We then obtain the restricted master of the column generation model (M1).

$$
\text { minimize } \quad c^{v e h} \cdot z+\sum_{v \in V} \sum_{k \in \hat{K}} p_{k} \cdot r_{v k}
$$


subject to

$$
\begin{array}{ccc}
{\left[\lambda_{m}\right]} & \sum_{v \in V} \sum_{k \in \hat{K}} a_{k m} \cdot r_{v k} \geq Q_{m} & m \in M \\
{\left[\gamma_{m d}\right]} & \sum_{v \in V} \sum_{k \in \hat{K}} g_{k m d} \cdot r_{v k} \leq N \cdot w_{m d} & m \in M, d \in D \\
\sum_{d \in D} w_{m d} \leq W_{m} & m \in M \\
{\left[\tau_{v d}\right]} & v \in V, d \in D \\
\sum_{k \in \hat{K}} h_{k d} \cdot r_{v k} \leq 1 & d \in D \\
\sum_{v \in V} \eta_{k \in \hat{K}} h_{k d} \cdot r_{v k} \leq z & v \in V, k \in \hat{K} \\
0 \leq r_{v k} \leq 1 & m \in M, d \in D
\end{array}
$$

Objective (17) minimizes the vehicle and route costs. Constraint set 18 models the demand constraints. Constraints (19) relate the $w_{m d}$ and route variables. Constraint set 20 imposes the maximal number of visits per customer. Additionally, a vehicle can only perform one route per day, as is ensured by constraints 21. Constraints 22 force $z$ to equal the minimal number of vehicles needed. Finally, constraints 23)-25 define the domains of the decision variables. The duals associated with constraints (18, (19), 21) and (22) are given between square brackets. These duals serve as input for the pricing problem (P1).

The parameters $p_{k}, a_{k m}, g_{k m d}$ and $h_{k d}$ obtain their values from the solution of the subproblem (P1), in which the following decision variables are used:

$g_{m d} \quad=1$ if customer $m$ is visited on day $d$; 0 otherwise

$h_{d} \quad=1$ if route is used on day $d ; 0$ otherwise

$y_{m}^{1} \quad=1$ if type 1 trip is to customer $m$; 0 otherwise

$y_{m}^{2} \quad$ number of type 2 trips to customer $m$

$q_{m}^{2} \quad=1$ if customer $m$ is visited by at least one type 2 trip, 0 otherwise [-]

$x_{m}^{1} \quad$ total load of type 1 trips to customer $m$ [tonne]

$x_{m}^{2} \quad$ total load of type 2 trips to customer $m$ [tonne]

Moreover, $c^{h} \cdot t_{m}^{1}$ and $c^{h} \cdot t_{m}^{2}$ are represented by $c_{m}^{1}$ and $c_{m}^{2}$, respectively. The subproblem (P1) to price out a new column is as follows:

$$
\begin{aligned}
\operatorname{minimize} & \sum_{m \in M}\left(c_{m}^{1} \cdot y_{m}^{1}+c_{m}^{2} \cdot y_{m}^{2}\right)-\sum_{m \in M} \lambda_{m} \cdot\left(x_{m}^{1}+x_{m}^{2}\right) \\
& -\sum_{m \in M} \sum_{d \in D} \gamma_{m d} \cdot g_{m d}-\sum_{v \in V} \sum_{d \in D} \tau_{v d} \cdot h_{d}-\sum_{d \in D} \eta_{d} \cdot h_{d}
\end{aligned}
$$


subject to

$$
\begin{aligned}
& x_{m}^{1} \leq L \cdot y_{m}^{1} \quad m \in M \\
& x_{m}^{2} \leq L \cdot y_{m}^{2} \quad m \in M \\
& x_{m}^{1}+x_{m}^{2} \leq Q_{m} \quad m \in M \\
& \sum_{m \in M}\left(s_{m} \cdot\left(x_{m}^{1}+x_{m}^{2}\right)+t_{m}^{1} \cdot y_{m}^{1}+t_{m}^{2} \cdot y_{m}^{2}\right) \leq T \\
& \sum_{m \in M} y_{m}^{1}=1 \\
& \sum_{d \in D} h_{d}=1 \\
& g_{m d}+1 \geq y_{m}^{1}+h_{d} \quad m \in M, d \in D \\
& N \cdot g_{m d}+1 \geq y_{m}^{2}+h_{d} \quad m \in M, d \in D \\
& g_{m d} \leq h_{d} \quad m \in M, d \in D \\
& g_{m d} \leq y_{m}^{1}+y_{m}^{2} \quad m \in M, d \in D \\
& \left(t_{m}^{\text {dep }}-t_{m}^{\text {disp }}\right)-N \cdot\left(1-y_{m}^{1}\right) \leq\left(t_{m^{\prime}}^{\text {dep }}-t_{m^{\prime}}^{\text {disp }}\right)+N \cdot\left(1-q_{m^{\prime}}^{2}\right) \quad m, m^{\prime} \in M \\
& N \cdot q_{m}^{2} \geq y_{m}^{2} \\
& q_{m}^{2} \leq y_{m}^{2} \\
& y_{m}^{1} \in\{0,1\} \\
& y_{m}^{2} \in\{0,1,2, \ldots\} \\
& q_{m}^{2} \in\{0,1\} \\
& x_{m}^{1}, x_{m}^{2} \geq 0 \\
& g_{m d} \in\{0,1\} \\
& h_{d} \in\{0,1\}
\end{aligned}
$$

The objective 26 minimizes the reduced cost. It consists of the real monetary cost of a route, corrected by the terms with the master's dual variables $\lambda_{m}, \gamma_{m d}$ and $\eta_{d}$. The first two sets of constraints 27)-28 ensure that the amount collected during a trip does not exceed the capacity of a vehicle, while constraints 29 make sure that collected amounts do not exceed a customer's demand. Constraint 30 restricts the duration of the route. Constraints (31) and (32) impose that the route contains exactly one type 1 trip and is designed for one specific day. Additionally, constraints (33)-36 enforce that if a customer $m$ is visited on day $d$ by this route, its corresponding $g_{m d}$-variable is forced to 1 . Constraints (37) make sure that the type 1 trip is performed to a customer that is relatively closer to the depot than to the disposal facility, as compared to other customers visited by vehicle $\mathrm{v}$ on day $\mathrm{d}$ on type 2 trips. This constraint is a valid inequality (Van Engeland, 2019). Constraints 38 - 39 make sure that the $q^{2}$-variables obtain correct values. Finally, constraints 40-45 define the domains of the decision variables.

The result of this pricing problem can be translated into a new column $k$ by copying the solution values to the corresponding parameters in the master problem: 


$$
\begin{array}{rll}
\sum_{m \in M}\left(c_{m}^{1} y_{m}^{1}+c_{m}^{2} y_{m}^{2}\right) & \rightarrow p_{k} & \\
\left(x_{m}^{1}+x_{m}^{2}\right) & \rightarrow a_{k m} \quad m \in M \\
g_{m d} & \rightarrow g_{k m d} \quad m \in M, d \in D \\
h_{d} & \rightarrow h_{k d} \quad d \in D
\end{array}
$$

Columns are added as long as they have a negative reduced cost. When the pricing problem results in a nonnegative reduced cost, an optimal LP solution is found and CG ends. To find an optimal integral solution, the CG procedure should be complemented with a branch-and-bound search giving rise to a branch-and-price algorithm. A possible branching scheme works as follows. If the column variable associated to route $k^{\prime}$ for day $d$ obtains a fractional value in the final master, there must be another route $k$ " for day $d$ which differs at least for one municipality $m$ in the waste to be collected, i.e. $a_{k^{\prime} m} \neq a_{k^{\prime \prime} m}$. In the master we can use a binary branching scheme in which we exclude all routes $k$ for day $d$ having $a_{k m} \leq a_{k^{\prime} m}$ while in the right branch we exclude all routes $k$ for day $d$ having $a_{k m} \geq a_{k^{\prime} m}+\epsilon$. In the pricing problem it suffices to add the corresponding constraints on $x_{m}^{1}+x_{m}^{2}$ if the route is generated for day $d$. Unfortunately, branching may lead to a large number of nodes where each node requires a CG procedure to find its optimal LP relaxation as new columns might price out after branching. Since the real-life problem entails tactical decision-making and our objective is already an approximation in which the routing component is ignored, finding an optimal solution does not warrant the extra computational effort. Therefore, we obtain an integral (heuristic) solution by solving the master with added integrality constraints (IM1) including only those columns generated during the CG phase of the root node.

\subsection{Start columns}

We initialize the set of start columns with one route for each customer. This route consists of a type 1 trip to the customer, which collects as much waste as possible. It respects the capacity, demand (i.e., no more waste than available at that customer is loaded) and working time constraint. However, the limited number of vehicles available might imply that there is no feasible schedule using only these routes. To guarantee feasibility, a "super column" is added that collects all waste of all customers. Of course this route is infeasible with respect to both capacity and work time constraints, hence a very high cost is assigned to it. In summary, the set of start columns contains $|M|+1$ columns or routes.

\subsection{Lower bound}

To evaluate the quality of the solutions retrieved in the heuristic column generation procedure, two calculation methods for a lower bound are proposed.

Since the last master LP (M1) is a relaxation of (IM1), its solution value can be used as a lower bound. This is the first lower bound, LB A.

The second lower bound can be obtained as follows. First, the objective function (17) in the column generation approach is changed in order to only minimize the number of vehicles, regardless of the cost of the routes.

(M1a) minimize $\quad z$

subject to 18$)$ - 25]. Also the pricing problem (P1) is changed: the first term in 26$)\left(\sum_{m \in M}\left(c_{m}^{1} \cdot y_{m}^{1}+\right.\right.$ $\left.\left.c_{m}^{2} \cdot y_{m}^{2}\right)\right)$ is removed. The objective of the last master LP (M1a) provides a fractional lower bound on the number of trucks needed. Note that solving the last master (M1a) with added integrality constraints on 
the column variables would lead to a heuristic solution and can, therefore, not be used as an LB. Instead, the obtained (fractional) solution is rounded to the upper integer to obtain the minimal number of vehicles needed. Afterwards, this is multiplied with the vehicle cost $c^{v e h}$ to obtain the minimal truck cost.

Second, the costs of the routes in the column generation process need to be minimized, regardless of the number of vehicles needed. Again the objective function (17) is altered:

$$
\text { minimize } \quad \sum_{v \in V} \sum_{k \in \hat{K}} p_{k} \cdot r_{v k}
$$

subject to (18)-25). The pricing problem (P1) remains unchanged, but $|V|$ should be sufficiently high as was described in Section 3. Again, the objective of the last master LP (M1b) provides a lower bound on the route cost. The lower bound B (LB B) is obtained by adding this (fractional) route cost to the minimal truck cost.

\subsection{A reduced variables MIP-based heuristic: the two trip heuristic}

For the test instances, all heuristic solutions of the column generation approach consist of routes with at most one type 2 trip (see Section 5.1). This was the inspiration for the two trip heuristic. All combinations with one or two trips (i.e., one type 1 and none or one type 2 trip) are constructed. Routes consisting solely out of one type 1 trip collect as much waste as possible from the visited customer, considering $L, T$ and $Q_{m}$. This amount is calculated as:

$$
\bar{x}_{m}^{1}=\min \left(Q_{m} ; L ; \frac{T-t_{m}^{1}}{s_{m}}\right) \quad m \in M
$$

In the routes composed of two trips, the type 1 trip is made to the customer $m$ which is relatively closest to the depot. The type 2 trip visits the customer $m^{\prime}$ relatively closer to the disposal facility. For each such combination, two variants of the route are made: one route which collects as much waste as possible (considering $L, T$ and $Q_{m}$ ) on the type 1 trip and uses the remaining time to collect the waste on the type 2 trip:

$$
\begin{array}{ll}
\bar{x}_{m}^{1}=\min \left(Q_{m} ; L ; \frac{T-t_{m}^{1}-t_{m^{\prime}}^{2}}{s_{m}}\right) & m, m^{\prime} \in M:\left(t_{m}^{d e p}-t_{m}^{d i s p}\right) \leq\left(t_{m^{\prime}}^{d e p}-t_{m^{\prime}}^{d i s p}\right) \quad \& \& \quad m^{\prime} \geq m \\
\bar{x}_{m^{\prime}}^{2}=\min \left(Q_{m^{\prime}} ; L ; \frac{T-t_{m}^{1}-t_{m^{\prime}}^{2}-\bar{x}_{m}^{1} \cdot s_{m}}{s_{m^{\prime}}}\right) & m, m^{\prime} \in M:\left(t_{m}^{d e p}-t_{m}^{d i s p}\right) \leq\left(t_{m^{\prime}}^{d e p}-t_{m^{\prime}}^{d i s p}\right) \quad \& \& \quad m^{\prime} \geq m
\end{array}
$$

The other variant of the route collects as much waste as possible (considering $L, T$ and $Q_{m}$ ) on the type 2 trip and uses the remaining time to collect the waste on the type 1 trip:

$$
\begin{array}{ll}
\bar{x}_{m^{\prime}}^{2}=\min \left(Q_{m^{\prime}} ; L ; \frac{T-t_{m}^{1}-t_{m^{\prime}}^{2}}{s_{m^{\prime}}}\right) & m, m^{\prime} \in M:\left(t_{m}^{d e p}-t_{m}^{d i s p}\right) \leq\left(t_{m^{\prime}}^{d e p}-t_{m^{\prime}}^{d i s p}\right) \quad \& \& \quad m^{\prime} \geq m \\
\bar{x}_{m}^{1}=\min \left(Q_{m} ; L ; \frac{T-t_{m}^{1}-t_{m^{\prime}}^{2}-\bar{x}_{m^{\prime}}^{2} \cdot s_{m}}{s_{m^{\prime}}}\right) & m, m^{\prime} \in M:\left(t_{m}^{d e p}-t_{m}^{d i s p}\right) \leq\left(t_{m^{\prime}}^{d e p}-t_{m^{\prime}}^{d i s p}\right) \quad \& \& \quad m^{\prime} \geq m
\end{array}
$$

Hence, a total of $|M|+|M| \cdot(|M|+1)$ routes are generated. After route construction, a MIP model similar to (IM1) decides on the day-route combinations. Note that this two trip heuristic (T1) is a simplification of the original problem (only two trips per route) and hence serves as a heuristic. The lower bounds of Section 4.3 will be used to evaluate the solutions. 
Table 1: Parameters of the instances. Note that the available number of vehicles $(|V|)$ was obtained as described in Section 3

\begin{tabular}{lcc}
\hline & instance 1 & instance 2 \\
\hline type of waste & plastic, metal, & paper and \\
& drink cartons (PMDa) & cardboard (PCa) \\
vehicle capacity $(L)$ & 2.0 tonnes & 8.8 tonnes \\
available vehicles $(|V|)$ & 8 & 15 \\
avg. demand per customer & 10 tonnes & 83 tonnes \\
$\#$ customers $(M)$ & 13 & 29 \\
available time $(T)$ & 7.5 hours & 7.5 hours \\
avg. duration $t_{m}^{1}$ & 1.3 hours & 1.8 hours \\
avg. duration $t_{m}^{2}$ & 1.0 hours & 1.4 hours \\
avg. collection speed & 2.11 h/tonne & 0.45 h/tonne \\
avg. unloading time & 0.25 h & $0.25 \mathrm{~h}$ \\
planning horizon $(D)$ & 10 days & 20 days \\
maximum number of visits $\left(W_{m}\right)$ & 3 & 3 \\
variable cost $\left(c^{h}\right)$ & 10 & 10 \\
fixed cost $\left(c^{\text {veh }}\right)$ & 100 & 100 \\
cost ratio $\left(c^{\text {veh }} / c^{h}\right)$ & 10 & 10 \\
\hline
\end{tabular}

\section{Computational Results}

In this section the CG approach (Section 4.1) and the two trip heuristic (Section 4.4) are applied to realistic test instances. The first instance concerns PMD collected every two weeks, i.e., a cycle period of 10 working days. The 13 customers represent 13 Flemish municipalities, which are located around a rather centralized positioned depot and disposal facility (see Figure 2). Instance 2 is about monthly (i.e., a cycle period of 20 working days) paper and cardboard (PC) collection in 29 Flemish municipalities. The depot is located centrally while the disposal facility is situated in the upper end of the area (see Figure 3). In both cases, an intermunicipal authority (IA) is responsible for collection of the waste flows. As required by the IAs, for both instances customers can be visited at most 3 times during the considered planning horizon, i.e., $W_{m}=3$. Details of these instances are given in Table1. An entire overview of the instance parameters can be found in Tables 6 and 7 in Appendix B. Additional test cases (labeled "a", see Section 5.4) are generated based on these two real-life instances. The results are discussed in the subsequent sections.

\subsection{Results $C G$ approach}

Recall that each column is generated for a specific day. To find higher quality solutions upon solving the final master (IM1) we added the day assignment again as a decision for each of the columns, i.e. each of the generated columns could be used for each day $d$. This increased decision dimension, however, combined with the complex min-max structure with respect to the number of vehicles (incorporated in the z-variables) makes solving the final master (IM1) to optimality (too) computational expensive. Therefore, we stop solving IM1 as soon as either a time limit of 120 seconds is reached or a solution within a MIP gap tolerance of $0.4 \%$ is found.

For the first real-life instance (PMDa), the CG approach found a heuristic solution of 1561.12 euro within 2 minutes 14 seconds. The schedule is presented in Table 2 and consists of 13 routes with only type 1 trips, and 9 routes with both a type 1 and a type 2 trip. Recall that routes define a combination of customers for a certain day. In our case of identical vehicles, these routes can be assigned to one or more of the available vehicles. The day with the largest number of routes will determine the number of vehicles needed. The last column of Table 2 shows that a fleet of 6 vehicles is needed. Type 2 trips are performed to customers 1,3 , 10 and 11. These customers are located relatively close to the disposal facility. Lower bound A and B were 1459.98 and 1472.89 , respectively, yielding gaps of circa $6.48 \%$ and $5.65 \%$. Figure 2 gives an illustration of this solution. Coloured customers are visited on a type 2 trip. Visiting a customer starting at the disposal 
facility (i.e., on a type 2 trip) can save driving hours. This is why these customers are more or less situated near the disposal facility.

Table 2: First instance, based on realistic data of PMD collection: schedule obtained with CG heuristic. A total of 6 vehicles is needed.

\begin{tabular}{|c|c|c|c|c|c|}
\hline day & $\begin{array}{c}\text { first } \\
\text { customer }\end{array}$ & $\begin{array}{c}\text { second } \\
\text { customer }\end{array}$ & $\begin{array}{c}\text { amount collected } \\
x^{1} \text { [tonne] }\end{array}$ & $\begin{array}{c}\text { amount collected } \\
x^{2} \text { [tonne] }\end{array}$ & $\begin{array}{l}\text { number of times } \\
\text { route is performed }\end{array}$ \\
\hline \multirow[t]{3}{*}{1} & 6 & & 2 & & 3 \\
\hline & 12 & 10 & 0.67 & 2 & 2 \\
\hline & 10 & & 2 & & 1 \\
\hline \multirow[t]{3}{*}{2} & 13 & & 2 & & 2 \\
\hline & 9 & & 2 & & 1 \\
\hline & 4 & 3 & 0.84 & 2 & 3 \\
\hline \multirow[t]{2}{*}{3} & 12 & 10 & 0.67 & 2 & 3 \\
\hline & 12 & 3 & 0.93 & 2 & 3 \\
\hline \multirow[t]{2}{*}{4} & 10 & & 2 & & 2 \\
\hline & 2 & & 2 & & 4 \\
\hline 5 & 2 & & 2 & & 6 \\
\hline \multirow[t]{2}{*}{6} & 7 & & 2 & & 3 \\
\hline & 13 & & 2 & & 3 \\
\hline 7 & 4 & 11 & 0.87 & 2 & 6 \\
\hline \multirow[t]{3}{*}{8} & 4 & & 2 & & 1 \\
\hline & 4 & 1 & 0.88 & 2 & 4 \\
\hline & 5 & 1 & 2 & 1.11 & 1 \\
\hline \multirow[t]{3}{*}{9} & 6 & & 2 & & 1 \\
\hline & 13 & 3 & 0.71 & 2 & 2 \\
\hline & 5 & 3 & 0.94 & 2 & 3 \\
\hline \multirow{2}{*}{10} & 8 & & 2 & & 4 \\
\hline & 9 & & 2 & & 2 \\
\hline
\end{tabular}

For the second real-life instance (PCa), a solution was found in 6.5 minutes. The total cost amounts to 6440.83 euro for the 20 day period. The gap with respect to LB A (6134.63) was stronger than that with respect to LB B (5941.10): $4.75 \%$ versus $7.75 \%$. A total of 13 vehicles was needed to perform the schedule. Customers visited on a type 2 trip were 1, 6, 9, 17, 19, 20 and 29, as indicated in Figure 3. Again, these customers are roughly located in the vicinity of the disposal facility. Detailed results are given in Table 3 .

\subsection{Results two trip heuristic}

This section discusses the results of the two trip heuristic (T1), as described in Section 4.4 . Since the two trip heuristic does not produce a lower bound that allows to calculate an optimality gap, we compare the solutions to the lower bounds LB A and LB B obtained by the CG approach. The results for different time limits are summarized in Table 4 A global time limit of 10 minutes, including time for the generation of the routes, is acceptable and gives good results. For the PMD case, the two trip heuristic found a solution of 1537.10 (gap of $4.36 \%$ with respect to LB B of 1472.89). In the case of PC, the total cost was 6355.00 (gap of $3.47 \%$ with respect to LB A of 6134.63 ).

\subsection{Results basic MIP with $N=1$}

As the 2 trip heuristic only allows routes of at most 2 trips, a similar performance could be expected from the basic MIP approach in which we allow for at most 2 municipalities visited per day per truck. To find out, we set the big-M (N) to 1 in constraint (7) of the basic MIP formulation F1 and test the instances PMDa and PKa. We stop the solver after 8000 seconds and compare with the results of the 2 trip heuristic (600 seconds) and CG algorithm (400 seconds). Table 5 reports the results.

For the smaller instance, PMDa, we see that the approach of solving the basic MIP with N=1 succeeds in finding a better solution, although the reported gap from MIP solving is still large (11.56\%). The solutions obtained by the 2 trip heuristic and CG approach are slightly worse, but the gaps obtained from LB A and LB B are much tighter than the MIP gap obtained by CPLEX in applying the basic MIP with N=1. 


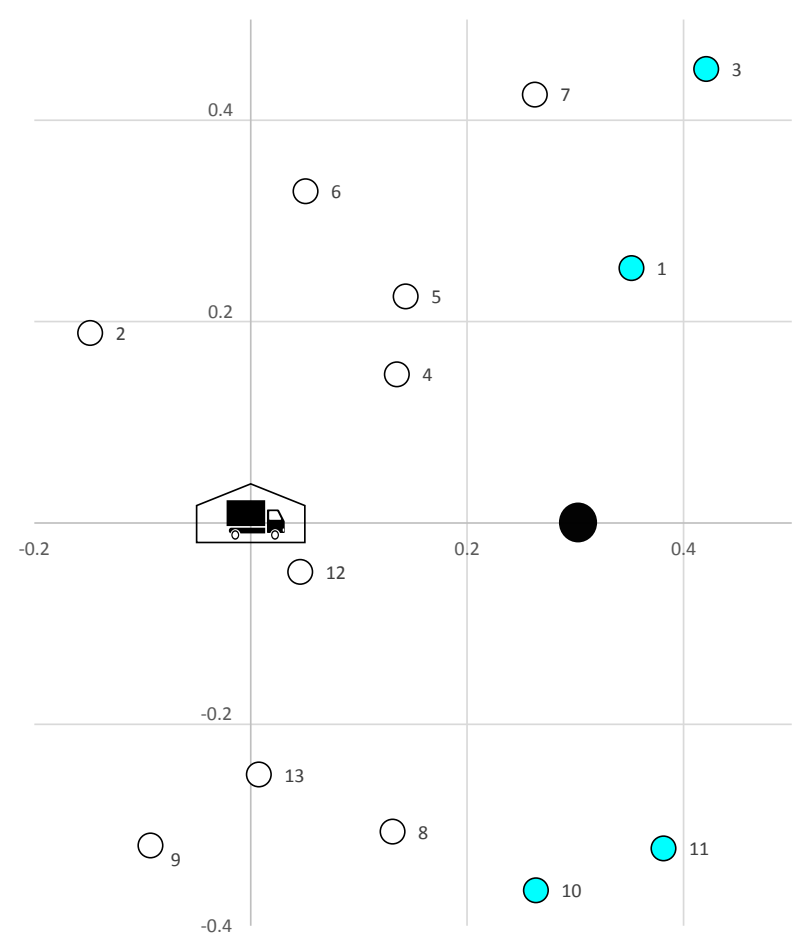

Figure 2: Schedule of first instance: coloured customers $(1,3,10,11)$ are visited on a type 2 trip.

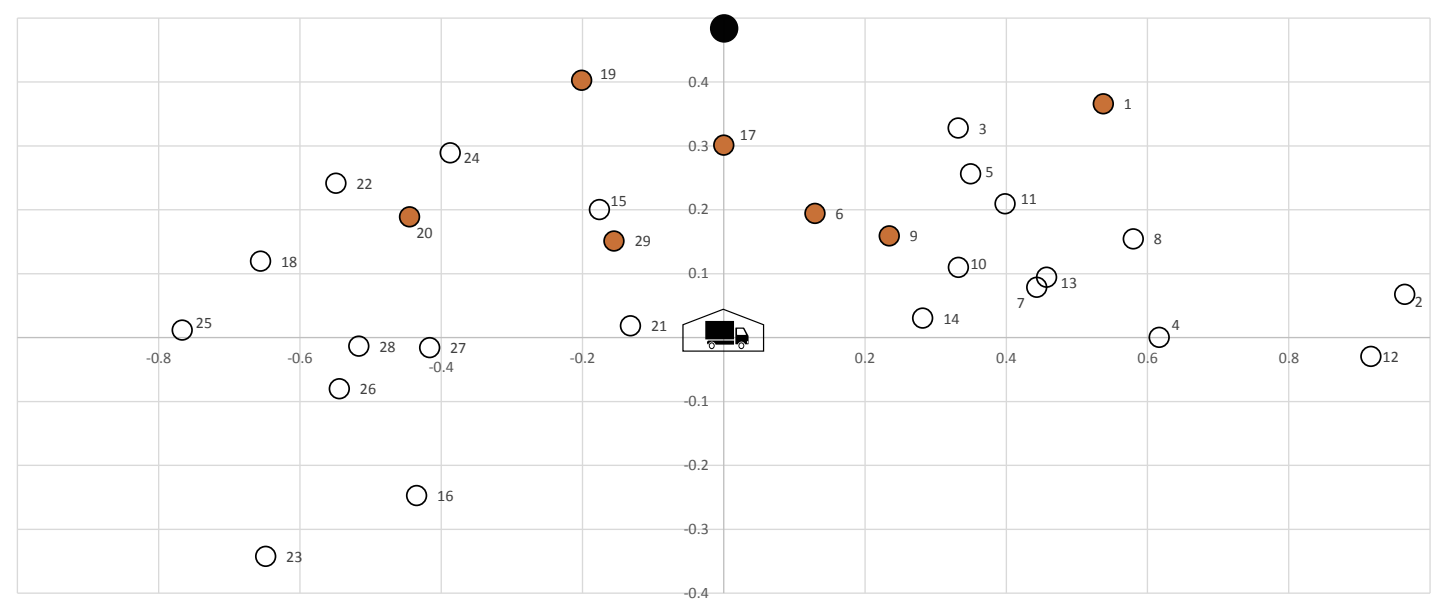

Figure 3: Schedule of second instance: customers 1, 6, 9, 17, 19, 20 and 29 are visited on a type 2 trip. 
Table 3: Second instance, based on realistic data of paper and cardboard collection: schedule obtained with CG heuristic. A total of 13 vehicles was needed.

\begin{tabular}{|c|c|c|c|c|c|}
\hline day & $\begin{array}{l}\text { first } \\
\text { customer }\end{array}$ & $\begin{array}{c}\text { second } \\
\text { customer }\end{array}$ & $\begin{array}{c}\text { amount collected } \\
x^{1}[\text { tonne] }\end{array}$ & $\begin{array}{c}\text { amount collected } \\
x^{2}[\text { tonne] }\end{array}$ & $\begin{array}{l}\text { number of times } \\
\text { route is performed }\end{array}$ \\
\hline \multirow[t]{5}{*}{1} & 2 & & 8.8 & & \\
\hline & 19 & & & & \\
\hline & 21 & 17 & 8.8 & 6.41 & 1 \\
\hline & 26 & 17 & 8.8 & 3.96 & 2 \\
\hline & 9 & 19 & 8.8 & 3.69 & \\
\hline \multirow[t]{2}{*}{2} & 12 & & 8.8 & & 1 \\
\hline & & 29 & $\begin{array}{l}8.8 \\
8.8\end{array}$ & 29 & 10 \\
\hline \multirow[t]{2}{*}{3} & $\begin{array}{r}29 \\
5\end{array}$ & & $\begin{array}{l}8.8 \\
8.8\end{array}$ & 2.92 & 3 \\
\hline & & 17 & 8.8 & 3.96 & 10 \\
\hline \multirow[t]{2}{*}{4} & 4 & 6 & 8.8 & 4.63 & 2 \\
\hline & ${ }_{6}^{6}$ & & $\begin{array}{l}8.8 \\
8.8\end{array}$ & & $\begin{array}{c}1 \\
10\end{array}$ \\
\hline \multirow[t]{4}{*}{5} & 7 & & $\begin{array}{l}8.0 \\
8.8\end{array}$ & & 3 \\
\hline & 19 & & 8.8 & & 2 \\
\hline & 29 & & 8.8 & & 1 \\
\hline & 9 & 19 & 8.8 & 3.69 & 7 \\
\hline \multirow[t]{2}{*}{6} & 13 & & 8.8 & & 2 \\
\hline & 8 & & 8.8 & & 1 \\
\hline \multirow{3}{*}{7} & $\begin{array}{l}8 \\
7\end{array}$ & & $\begin{array}{l}8.8 \\
8.8\end{array}$ & & 10 \\
\hline & 10 & & $\begin{array}{l}8.8 \\
8.8\end{array}$ & & $\begin{array}{l}3 \\
4\end{array}$ \\
\hline & 22 & & 8.8 & & 6 \\
\hline \multirow[t]{4}{*}{8} & 14 & & 8.8 & & 5 \\
\hline & 22 & & 8.8 & & 2 \\
\hline & 21 & 9 & $\begin{array}{l}8.8 \\
8.8\end{array}$ & 6.87 & $\begin{array}{l}1 \\
3\end{array}$ \\
\hline & 21 & 1 & 5.78 & 8.8 & 2 \\
\hline \multirow[t]{3}{*}{9} & 5 & & 8.8 & & 2 \\
\hline & 11 & & 8.8 & & 7 \\
\hline & 25 & & 8.8 & & 4 \\
\hline \multirow{2}{*}{10} & 16 & & 8.8 & & 2 \\
\hline & $\begin{array}{l}23 \\
27\end{array}$ & & 8.8 & & 8 \\
\hline \multirow[t]{2}{*}{11} & 16 & & $\begin{array}{l}8.8 \\
8.8\end{array}$ & & $\begin{array}{l}3 \\
3\end{array}$ \\
\hline & 6 & & 8.8 & & 10 \\
\hline 12 & 8 & & 8.8 & & 7 \\
\hline \multirow{2}{*}{13} & 18 & & 8.8 & & 6 \\
\hline & 20 & & $\begin{array}{l}8.8 \\
8.8\end{array}$ & & 10 \\
\hline \multirow{4}{*}{14} & 2 & & $\begin{array}{l}8.8 \\
8.8\end{array}$ & & $\begin{array}{l}3 \\
1\end{array}$ \\
\hline & 16 & & 8.8 & & 6 \\
\hline & 6 & 1 & 3.99 & 8.8 & 4 \\
\hline & 4 & & 8.8 & 4.63 & 1 \\
\hline \multirow{2}{*}{15} & 4 & 20 & 8.8 & 4.17 & \\
\hline & 15 & & 8.8 & & 8 \\
\hline \multirow[t]{3}{*}{16} & & & $\begin{array}{l}8.8 \\
8.8\end{array}$ & & \\
\hline & $\begin{array}{l}13 \\
21\end{array}$ & 19 & $\begin{array}{l}8.8 \\
8.8\end{array}$ & & $\begin{array}{l}3 \\
2\end{array}$ \\
\hline & 4 & 19 & 8.8 & 3.83 & 8 \\
\hline 17 & 3 & & 8.8 & & 8 \\
\hline & 8 & & 8.8 & & 5 \\
\hline \multirow{2}{*}{18} & 12 & & 8.8 & & 10 \\
\hline & $\begin{array}{l}13 \\
25\end{array}$ & & $\begin{array}{l}8.8 \\
8.8\end{array}$ & & $\begin{array}{l}1 \\
2\end{array}$ \\
\hline 19 & 26 & & 8.8 & & 10 \\
\hline \multirow{3}{*}{20} & 26 & & 8.8 & & 3 \\
\hline & 12 & & 8.8 & & 1 \\
\hline & $\begin{array}{l}14 \\
28\end{array}$ & & $\begin{array}{l}8.8 \\
8.8\end{array}$ & & $\begin{array}{l}4 \\
8\end{array}$ \\
\hline
\end{tabular}

Table 4: Overview of the results.

\begin{tabular}{lrr}
\multicolumn{3}{c}{ Table 4: Overview of the results. } \\
\hline & instance 1 (PMDa) & instance 2 (PCa) \\
\hline LB A [euro] & 1459.98 & 6134.63 \\
LB B [euro] & 1472.89 & 5941.10 \\
CG solutions [euro] & 1561.12 & 6440.83 \\
CG \# routes generated [-] & 36 & 52 \\
best gap CG [\%] & 5.65 & 4.75 \\
2 trip MIP solutions (3 min) [euro] & 1537.10 & 6358.17 \\
2 trip MIP solutions (10 min) [euro] & 1537.10 & 6355.00 \\
2 trip MIP solutions (60 min) [euro] & 1537.10 & 6355.00 \\
best gap 2 trip MIP (10 min) [\%] & 4.36 & 3.47 \\
\hline
\end{tabular}

Table 5: Comparison basic MIP with $\mathrm{N}=1$.

\begin{tabular}{lrrr}
\hline & Basic MIP (N=1) & 2 trip & CG \\
& Best obj. value (MIP gap) & Best obj. Value (gap, LB) & Best obj. Value (gap, LB) \\
PMDa & $1515.3305(11.56 \%)$ & $1537.10(4.36 \%$, LB B $)$ & $1561.12(5.65 \%$, LB B) \\
PKa & $7616.0000(25.47 \%)$ & $6355.00(3.47 \%$, LB A $)$ & $6440.83(4.75 \%$, LB A) \\
\hline
\end{tabular}


For the larger instance, PKa, although the solution space is much smaller now, the new MIP did not succeed in finding the same quality solutions as the ones found by the 2 trip heuristic and CG approach. Note that providing the trips as decision variables (as is done in the 2 trip heuristic and CG approach) instead of the individual customer visits (as is done in the basic MIP formulation) leads to a formulation which achieves a much tighter approximation of the integer convex hull. As a consequence the LP relaxations of the nodes in the branch-and-bound tree are much tighter which enables a more efficient search as nodes can be pruned in an earlier stage based on bound comparisons. For larger instances this has a significant impact.

\subsection{Results other test instances}

To generate additional test instances, we varied the waste volumes $Q_{m}$ (instances indicated with $\mathrm{b}$, c and d) and travel times $t_{m}^{d e p}, t_{m}^{d i s p}$ and $t^{d i s p, d e p}$ (instances indicated with .a, .b, .c and .d). Details on these instances can be found in Appendix C. The results are summarized in Figure 4 All results are normalized with respect to the best bound:

$$
\text { normalized_result }=\frac{\text { result }}{\max (L B A, L B B)}
$$

The solutions presented in Figure 4 of the CG and two trip approach are of good quality when compared to the strongest lower bound. For the first class of instances (PMD), the solutions obtained by CPLEX are superior to the ones obtained by CG or the two trip heuristic. These are the smaller instances, with a shorter planning horizon. Recall that the bounds obtained by CG are needed to evaluate the quality of these solutions. The CG and two trip approach outperformed CPLEX in all the PC instances. For instance PC.d, CPLEX did not even find a feasible solution within 2 hours. Additionally, the figure shows that the results for the PC instances are better, i.e., they have lower gaps compared to those of the PMD instances. A first explanation for this are the larger objective values. Another reason might be that the planning horizon for the PC scenarios is longer, allowing for more flexibility and hence better results. As shown in Figure 4, for most instances the two trip heuristic proved to be superior to the CG approach. However, the CG approach was needed in order to obtain LB A and LB B.

Using a Friedman test with aligned ranks the results of the CG approach and the 2 trip heuristic for both groups of instances (PMD and PK) were compared with the general purpose solver CPLEX given a time limit of 2 hours. For the PMD instances, the null hypothesis that the two trip heuristic performs the same as CPLEX in terms of solution quality could not be rejected at a significance level of 0.05. At the same significance level, the test indicated that the CG approach differs from CPLEX on the PMD instances. Although CG performs worse than CPLEX, CG was still necessary to provide the lower bounds. For the PC instances, the statistical test revealed that both the $\mathrm{CG}$ and the two trip heuristic gave significant better results than CPLEX at the 0.05 level.

\section{Conclusion}

This paper proposes two heuristic solution procedure to obtain tactical waste collection schemes. The goal is to find a set of routes for each vehicle that satisfy both the demand and the frequency constraints and minimize the sum of the costs of all routes. After every customer, the central disposal facility has to be visited to weigh and discharge the waste. Each day of the planning horizon vehicles start and end their route at a depot. We only consider one depot and one disposal facility. Moreover, it was assumed that all vehicles 


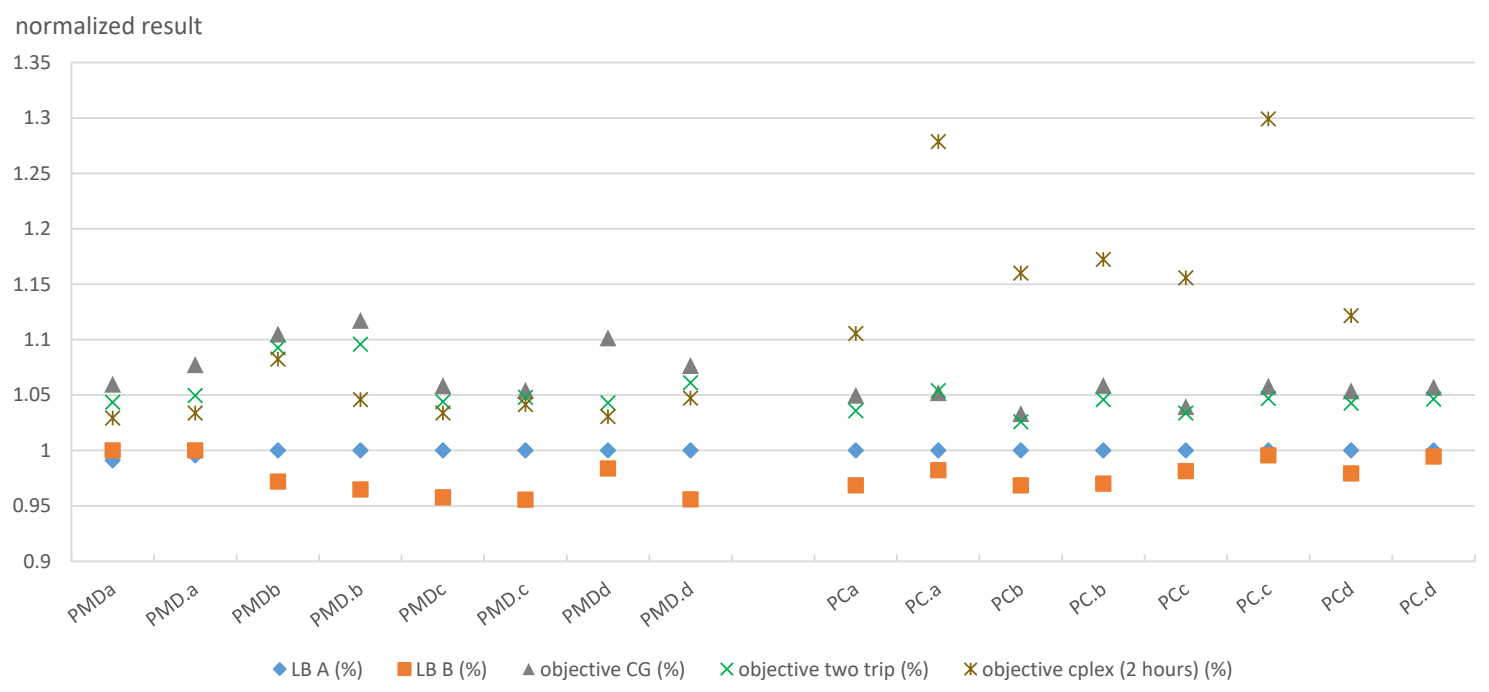

Figure 4: Overview of the solutions of the CG and two trip heuristics. All results are normalized with respect to the best bound. PMDa and PCa represent the initial cases discussed in the previous sections.

and days were identical.

A state-of-the-art solver could not solve the standard MIP formulation of two test problems to optimality, nor find a solution with an acceptable gap within a reasonable time limit. For this reason a heuristic column generation approach was proposed. This solution strategy yielded good solutions in an acceptable computation time. Type 2 trips proved to be useful and were implemented in all test instances. These trips are preferably made to customers closely located to the disposal facility. The 2 trip heuristic was inspired by the fact that the CG approach only generated routes with at most one type 2 trip. Moreover, the CG approach allowed to compute two lower bounds that proved to be much better than the LP relaxation bounds provided by a commercial MIP solver.

Our computational experiments reveal that the CG approach and the 2 trip heuristic outperform the basic MIP approach in terms of solution quality (at the significance level of 0.05) for large instances. For small instances, the basic MIP performs better, but the quality guarantee (measured as the MIP gap) is much worse than the quality guarantee provided by the two lower bounds based on CG. For all test instances, the combination of the 2 trip heuristic with the lower bounds of the column generation approach proved successful: good quality solution were obtained in acceptable computation time.

As an extension for future research, the solution strategy of the column generation approach could be improved by branching after each column generation phase. This would yield a branch-and-price algorithm which would give an optimal integer solution. A second direction could involve the possibility for working overtime. In this paper, the test instances were based on realistic data, and presume a constant number of working hours per day. Nevertheless, when the available time per day rises, more trips per route might be feasible, reducing total cost. Preliminary tests indicated that this is indeed the case. Hence, a direction for future research is the incorporation of overtime. This would introduce the option to work longer on one day and stop earlier on another. Finally, the consideration of external costs could be a fruitful avenue for future research. Not only the monetary costs of collection, but also external costs like, e.g. air pollution or noise, could be incorporated in the objective function. 


\section{References}

Andrea Arribas, C., Alejandra Blazquez, C., \& Lamas, A. (2010). Urban solid waste collection system using mathematical modelling and tools of geographic information systems. Waste Management \& Research, 28, 355-363. doi:10.1177/0734242X09353435.

Angelelli, E., \& Speranza, M. G. (2002). The application of a vehicle routing model to a waste-collection problem: two case studies. Journal of the Operational Research Society, 53, 944-952. URL: http://link. springer.com/10.1057/palgrave.jors.2601402 doi 10.1057/palgrave.jors.2601402.

Baldacci, R., Bartolini, E., Mingozzi, A., \& Valletta, A. (2011). An Exact Algorithm for the Period Routing Problem. Operations Research, 59, 228-241. URL: http://pubsonline.informs.org/doi/abs/10. 1287/opre.1100.0875, doi 10.1287/opre.1100.0875.

Bartodziej, P., Derigs, U., Malcherek, D., \& Vogel, U. (2009). Models and algorithms for solving combined vehicle and crew scheduling problems with rest constraints : an application to road feeder service planning in air cargo transportation. OR Spectrum, 31, 405-429. URL: https://doi.org/10.1007/ s00291-007-0110-7, doi $10.1007 / \mathrm{s} 00291-007-0110-7$.

Beliën, J., De Boeck, L., \& Van Ackere, J. (2014). Municipal Solid Waste Collection and Management Problems: A Literature Review. Transportation Science, 48, 78-102. URL: http://pubsonline.informs. org/doi/abs/10.1287/trsc.1120.0448, doi:10.1287/trsc.1120.0448

Bish, D. R. (2011). Planning for a bus-based evacuation. OR Spectrum, 33, 629-654. URL: https://doi. org/10.1007/s00291-011-0256-1. doi:10.1007/s00291-011-0256-1.

le Blanc, I., van Krieken, M., Krikke, H., \& Fleuren, H. (2006). Vehicle routing concepts in the closed-loop container network of ARN-a case study. OR Spectrum, 28, 53-71. URL: http://link.springer.com/ 10.1007/s00291-005-0003-6. doi 10.1007/s00291-005-0003-6.

Campbell, A. M., \& Wilson, J. H. (2014). Forty years of periodic vehicle routing. Networks, 63, 2-15. URL: http://onlinelibrary.wiley.com.kuleuven.ezproxy.kuleuven.be/doi/10.1002/net.21527/ abstract, doi:10.1002/net.21527.

Constantino, M., Gouveia, L., Mourão, M. C., \& Nunes, A. C. (2015). The mixed capacitated arc routing problem with non-overlapping routes. European Journal of Operational Research, 244, 445456. URL: http://www.sciencedirect.com/science/article/pii/S0377221715000624. doi:10.1016/ j.ejor.2015.01.042.

Cortinhal, M. J., Mourão, M. C., \& Nunes, A. C. (2016). Local search heuristics for sectoring routing in a household waste collection context. European Journal of Operational Research, 255, 6879. URL: http://www.sciencedirect.com/science/article/pii/S0377221716302326. doi:10.1016/ j.ejor.2016.04.013.

Costa, L., Contardo, C., \& Desaulniers, G. (2019). Exact branch-price-and-cut algorithms for vehicle routing. Transportation Science, 53, 946-985. URL: https://doi.org/10.1287/trsc.2018.0878, doi:10.1287/ trsc.2018.0878, arXiv:https://doi.org/10.1287/trsc.2018.0878.

Desaulniers, G., Desrosiers, J., \& Solomon, M. (2005). Column generation. Springer. 
European Commission (2017). Circular Economy Strategy. URL: http://ec.europa.eu/environment/ circular-economy/.

Francis, P., Smilowitz, K., \& Tzur, M. (2006). The period vehicle routing problem with service choice. Transportation Science, 40, 439-454. URL: http://pubsonline.informs.org/doi/abs/10.1287/trsc. 1050.0140

Ghiani, G., Guerriero, E., Manni, A., Manni, E., \& Potenza, A. (2013). Simultaneous personnel and vehicle shift scheduling in the waste management sector. Waste Management, 33, 1589-1594. URL: http://www . sciencedirect.com/science/article/pii/S0956053X13001694 doi:10.1016/j.wasman.2013.04.001

Ghiani, G., Laganà, D., Manni, E., Musmanno, R., \& Vigo, D. (2014). Operations research in solid waste management: A survey of strategic and tactical issues. Computers 83 Operations Research, 44, 2232. URL: http://www.sciencedirect.com/science/article/pii/S0305054813003018. doi:10.1016/ j.cor.2013.10.006.

Gomes Salema, M. I., Barbosa Povoa, A. P., \& Novais, A. Q. (2009). A strategic and tactical model for closed-loop supply chains. Or Spectrum, 31, 573-599. doi 10.1007/s00291-008-0160-5

Hernandez, F., Gendreau, M., \& Potvin, J.-Y. (2017). Heuristics for tactical time slot management: a periodic vehicle routing problem view: F. Hernandez et al. International Transactions in Operational Research, 24, 1233-1252. URL: http://doi.wiley.com/10.1111/itor.12403, doi:10.1111/itor.12403.

Ignall, E., Kolesar, P., \& Walker, W. (1972). Linear Programming Models of Crew Assignments for Refuse Collection. IEEE Transactions on Systems, Man, and Cybernetics, SMC-2, 664-666. doi:10.1109/TSMC. 1972.4309195.

Jang, W., Lim, H. H., Crowe, T. J., Raskin, G., \& Perkins, T. E. (2006). The Missouri Lottery Optimizes Its Scheduling and Routing to Improve Efficiency and Balance. Interfaces, 36, 302-313. URL: http:// pubsonline.informs.org/doi/abs/10.1287/inte.1060.0204 doi:10.1287/inte.1060.0204. Bibtex: jang_missouri_2006.

Kim, B.-I., Kim, S., \& Sahoo, S. (2006). Waste collection vehicle routing problem with time windows. Computers \& Operations Research, 33, 3624-3642. URL: http://www.sciencedirect.com/science/ article/pii/S0305054805001322. doi $10.1016 / j . c o r .2005 .02 .045$

Lübbecke, M. E. (2011). Column generation. In Wiley Encyclopedia of Operations Research and Management Science. American Cancer Society. URL: https://onlinelibrary.wiley. com/doi/abs/10.1002/9780470400531.eorms0158. doi 10.1002/9780470400531.eorms0158 arXiv:https://onlinelibrary.wiley.com/doi/pdf/10.1002/9780470400531.eorms0158

List, G. F., Wood, B., Turnquist, M. A., Nozick, L. K., Jones, D. A., \& Lawton, C. R. (2006). Logistics planning under uncertainty for disposition of radioactive wastes. Computers $\&$ Operations Research, 33, 701723. URL: http://www.sciencedirect.com/science/article/pii/S0305054804001807, doi:10.1016/ j.cor.2004.07.017.

Mansini, R., \& Speranza, M. G. (1998). A linear programming model for the separate refuse collection service. Computers $\& 3$ Operations Research, 25, 659-673. URL: http://www.sciencedirect.com/science/ article/pii/S0305054897000944. doi 10.1016/S0305-0548(97)00094-4. 
Mourão, M. C., Nunes, A. C., \& Prins, C. (2009). Heuristic methods for the sectoring arc routing problem. European Journal of Operational Research, 196, 856-868. doi 10.1016/j.ejor.2008.04.025 WOS:000263817600002.

Ramos, T. R. P., \& Oliveira, R. C. (2011). Delimitation of service areas in reverse logistics networks with multiple depots. Journal of the Operational Research Society, 62, 1198-1210. doi:10.1057/jors.2010.83 WOS:000290258800003.

Toth, P., Vigo, D., Toth, P., \& Vigo, D. (2014). Vehicle Routing: Problems, Methods, and Applications, Second Edition. Philadelphia, PA, USA: Society for Industrial and Applied Mathematics.

Van Engeland, J. (2019). Optimization approaches for recyclable municipal solid waste collection. Ph.D. thesis KU Leuven, Faculty of Economics and Business.

\section{Appendix A: IP model to find fleet size}

The following IP is solved to obtain the maximal fleet size $|V|$ used throughout the paper. It minimizes the number of vehicles needed, if collection was performed using only type 1 trips. The model was able to find an optimal solution for all test instances within seconds.

minimize $z$

subject to

$$
\begin{array}{cr}
x_{v m d}^{1} \leq L \cdot y_{v m d}^{1} & v \in V, m \in M, d \in D \\
\sum_{v \in V} \sum_{d \in D} x_{v m d}^{1}=Q_{m} & m \in M \\
\sum_{m \in M}\left(s_{m}\left(x_{v m d}^{1}\right)+t_{m}^{1} y_{v m d}^{1}\right) \leq T & v \in V, d \in D \\
\sum_{m \in M} y_{v m d}^{1} \leq 1 & v \in V, d \in D \\
z \geq \sum_{v \in V} \sum_{m \in M} y_{v m d}^{1} & v \in D, m \in M, d \in D \\
\sum_{m d} \geq y_{v m d}^{1} & m \in M \\
\sum_{d \in D} w_{m d} \leq W_{m} & v \in V, m \in M, d \in D \\
y_{v m d}^{1} \in\{0,1\} & v \in V, m \in M, d \in D \\
x_{v m d}^{1} \geq 0 & \\
w_{m d} \in\{0,1\} & m \in M, d \in D \\
z \in\{0,1,2, \ldots\} &
\end{array}
$$

For the interpretation of the constraints, we refer to Section 3 


\section{Appendix B: Initial instances}

This appendix presents the data on the initial instances (Table 6 and Table 7). Note that $t_{m}^{1}$ represents time for a type 1 trip to a given customer, excluding the time to perform collection at this customer. This time thus represents the aggregated time to drive from the depot to the customer, from the the customer to the disposal facility, the time to unload the waste and the time to drive from the disposal facility to the depot (at the end of the day). This should be multiplied with $c^{h}$ to obtain the cost of a type 1 trip. Similarly, $t_{m}^{2}$ represents the time needed for a type 2 trip to a given customer. The total time of such a type 2 trip is the time to drive from the disposal facility to the customer and back, and the time to unload the waste.

\begin{tabular}{ccccccc|cc}
\multicolumn{7}{c}{ Table 6: Instance 1 (PMDa): data. } \\
\hline customer & $t_{m}^{\text {deep }}$ & $t_{m}^{\text {disp }}$ & $t_{m}^{\text {disp,dep }}$ & $t_{m}^{\text {unl }}$ & $t_{m}^{1}$ & $t_{m}^{2}$ & $s_{m}$ & $Q_{m}$ \\
\hline 1 & 0.43 & 0.26 & 0.30 & 0.25 & 1.24 & 0.77 & 1.97 & 9.04 \\
2 & 0.24 & 0.49 & 0.30 & 0.25 & 1.28 & 1.22 & 2.22 & 19.23 \\
3 & 0.62 & 0.47 & 0.30 & 0.25 & 1.63 & 1.18 & 1.80 & 21.54 \\
4 & 0.20 & 0.22 & 0.30 & 0.25 & 0.97 & 0.69 & 2.07 & 13.27 \\
5 & 0.27 & 0.27 & 0.30 & 0.25 & 1.09 & 0.80 & 1.73 & 4.54 \\
6 & 0.33 & 0.41 & 0.30 & 0.25 & 1.30 & 1.08 & 2.68 & 6.35 \\
7 & 0.50 & 0.43 & 0.30 & 0.25 & 1.48 & 1.10 & 2.17 & 5.73 \\
8 & 0.33 & 0.35 & 0.30 & 0.25 & 1.23 & 0.95 & 2.30 & 6.38 \\
9 & 0.33 & 0.51 & 0.30 & 0.25 & 1.39 & 1.26 & 2.26 & 4.88 \\
10 & 0.45 & 0.37 & 0.30 & 0.25 & 1.37 & 0.98 & 2.16 & 15.08 \\
11 & 0.50 & 0.33 & 0.30 & 0.25 & 1.38 & 0.92 & 1.90 & 11.38 \\
12 & 0.07 & 0.26 & 0.30 & 0.25 & 0.88 & 0.77 & 1.98 & 5.62 \\
13 & 0.25 & 0.38 & 0.30 & 0.25 & 1.18 & 1.02 & 2.15 & 11.31 \\
\hline
\end{tabular}

Table 7: Instance 2 (PCa): data.

\begin{tabular}{ccccccc|cc}
\multicolumn{7}{c}{ Table $7:$ Instance $2(\mathrm{PCa}):$ data. } \\
\hline custome & $t_{m}^{\text {dep }}$ & $t_{m}^{\text {disp }}$ & $t_{m}^{\text {disp } \text { dep }}$ & $t_{m}^{\text {unl }}$ & $t_{m}^{1}$ & $t_{m}^{2}$ & $s m$ & $Q m$ \\
\hline 1 & 0.65 & 0.55 & 0.48 & 0.25 & 1.93 & 1.35 & 0.35 & 44.17 \\
2 & 0.97 & 1.05 & 0.48 & 0.25 & 2.75 & 2.35 & 0.52 & 13.75 \\
3 & 0.47 & 0.37 & 0.48 & 0.25 & 1.57 & 0.98 & 0.48 & 67.92 \\
4 & 0.62 & 0.78 & 0.48 & 0.25 & 2.13 & 1.82 & 0.28 & 98.33 \\
5 & 0.43 & 0.42 & 0.48 & 0.25 & 1.58 & 1.08 & 0.48 & 42.50 \\
6 & 0.23 & 0.32 & 0.48 & 0.25 & 1.28 & 0.88 & 0.44 & 214.17 \\
7 & 0.45 & 0.60 & 0.48 & 0.25 & 1.78 & 1.45 & 0.47 & 45.83 \\
8 & 0.60 & 0.67 & 0.48 & 0.25 & 2.00 & 1.58 & 0.39 & 198.33 \\
9 & 0.28 & 0.40 & 0.48 & 0.25 & 1.42 & 1.05 & 0.37 & 152.50 \\
10 & 0.35 & 0.50 & 0.48 & 0.25 & 1.58 & 1.25 & 0.52 & 31.33 \\
11 & 0.45 & 0.48 & 0.48 & 0.25 & 1.67 & 1.22 & 0.65 & 59.83 \\
12 & 0.92 & 1.05 & 0.48 & 0.25 & 2.70 & 2.35 & 0.50 & 100.83 \\
13 & 0.47 & 0.60 & 0.48 & 0.25 & 1.80 & 1.45 & 0.47 & 49.17 \\
14 & 0.28 & 0.53 & 0.48 & 0.25 & 1.55 & 1.32 & 0.52 & 75.00 \\
15 & 0.27 & 0.33 & 0.48 & 0.25 & 1.33 & 0.92 & 0.49 & 64.67 \\
16 & 0.50 & 0.85 & 0.48 & 0.25 & 2.08 & 1.95 & 0.43 & 89.17 \\
17 & 0.27 & 0.12 & 0.48 & 0.25 & 1.12 & 0.48 & 0.45 & 53.33 \\
18 & 0.67 & 0.75 & 0.48 & 0.25 & 2.15 & 1.75 & 0.47 & 48.58 \\
19 & 0.45 & 0.22 & 0.48 & 0.25 & 1.40 & 0.68 & 0.58 & 122.00 \\
20 & 0.48 & 0.53 & 0.48 & 0.25 & 1.75 & 1.32 & 0.38 & 117.92 \\
21 & 0.13 & 0.48 & 0.48 & 0.25 & 1.35 & 1.22 & 0.29 & 62.50 \\
22 & 0.60 & 0.60 & 0.48 & 0.25 & 1.93 & 1.45 & 0.41 & 69.17 \\
23 & 0.73 & 1.05 & 0.48 & 0.25 & 2.52 & 2.35 & 0.51 & 68.83 \\
24 & 0.48 & 0.43 & 0.48 & 0.25 & 1.65 & 1.12 & 0.54 & 40.00 \\
25 & 0.77 & 0.90 & 0.48 & 0.25 & 2.40 & 2.05 & 0.40 & 44.17 \\
26 & 0.55 & 0.78 & 0.48 & 0.25 & 2.07 & 1.82 & 0.34 & 216.67 \\
27 & 0.42 & 0.65 & 0.48 & 0.25 & 1.80 & 1.55 & 0.37 & 27.92 \\
28 & 0.52 & 0.72 & 0.48 & 0.25 & 1.97 & 1.68 & 0.37 & 69.17 \\
29 & 0.22 & 0.37 & 0.48 & 0.25 & 1.32 & 0.98 & 0.44 & 117.50 \\
\hline
\end{tabular}

\section{Appendix C: other test instances}

This section presents the data on the other test instances (Tables 8 to 21). Note that $T_{i}^{1}$ represents the time for a type 1 trip to a given customer, excluding the time to perform collection at this customer. This time thus represents the aggregated time to drive from the depot to the customer, from the the customer to the disposal facility, the time to unload the waste and the time to drive from the disposal facility to the depot (at the end of the day). This should be multiplied with $C^{\text {hour }}$ to obtain the cost of a type 1 trip. Similarly, $T_{i}^{2}$ represents the time needed for a type 2 trip to a given customer. The total time of such a type 2 trip is the time to drive from the disposal facility to the customer and back, and the time to unload the waste. 
Additional instances (suffixes .a, b, .b, c, .c, d and .d) were generated based on the initial cases (suffixes a). We varied the waste volumes $Q_{i}$ (instances indicated with $\mathrm{b}, \mathrm{c}$ and d) and travel times $T_{i}^{\text {dep }}, T_{i}^{\text {disp }}$ and

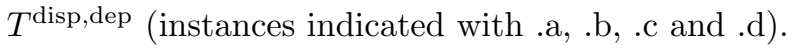

Table 8: Instance PMDb: data.

\begin{tabular}{ccccccc|cc} 
& \multicolumn{2}{c}{ Table 8: Instance PMDb: data. } \\
customer & $T_{i}^{\text {dep }}$ & $T_{i}^{\text {disp }}$ & $T_{i}^{\text {disp,dep }}$ & $T_{i}^{\text {unl }}$ & $T_{i}^{1}$ & $T_{i}^{2}$ & $S_{i}$ & $Q_{i}$ \\
\hline 1 & 0.43 & 0.26 & 0.30 & 0.25 & 1.24 & 0.77 & 1.97 & 10.96 \\
2 & 0.24 & 0.30 & 0.30 & 0.25 & 1.09 & 0.86 & 2.22 & 8.21 \\
3 & 0.62 & 0.47 & 0.30 & 0.25 & 1.63 & 1.18 & 1.80 & 18.90 \\
4 & 0.20 & 0.22 & 0.30 & 0.25 & 0.97 & 0.69 & 2.07 & 3.30 \\
5 & 0.27 & 0.27 & 0.30 & 0.25 & 1.09 & 0.80 & 1.73 & 2.30 \\
6 & 0.33 & 0.41 & 0.30 & 0.25 & 1.30 & 1.08 & 2.68 & 17.37 \\
7 & 0.50 & 0.43 & 0.30 & 0.25 & 1.48 & 1.10 & 2.17 & 8.40 \\
8 & 0.33 & 0.35 & 0.30 & 0.25 & 1.23 & 0.95 & 2.30 & 3.06 \\
9 & 0.33 & 0.32 & 0.30 & 0.25 & 1.20 & 0.88 & 2.26 & 6.46 \\
10 & 0.45 & 0.37 & 0.30 & 0.25 & 1.37 & 0.98 & 2.16 & 8.12 \\
11 & 0.50 & 0.33 & 0.30 & 0.25 & 1.38 & 0.92 & 1.90 & 19.39 \\
12 & 0.07 & 0.26 & 0.30 & 0.25 & 0.88 & 0.77 & 1.98 & 13.64 \\
13 & 0.25 & 0.38 & 0.30 & 0.25 & 1.18 & 1.02 & 2.15 & 6.48 \\
\hline
\end{tabular}

\begin{tabular}{ccccccc|cc} 
& \multicolumn{7}{c}{ Table $9:$ Instance PMDc: data. } \\
customer & $T_{i}^{\text {dep }}$ & $T_{i}^{\text {disp }}$ & $T_{i}^{\text {disp,dep }}$ & $T_{i}^{\text {unl }}$ & $T_{i}^{1}$ & $T_{i}^{2}$ & $S_{i}$ & $Q_{i}$ \\
\hline 1 & 0.43 & 0.26 & 0.30 & 0.25 & 1.24 & 0.77 & 1.97 & 21.28 \\
2 & 0.24 & 0.30 & 0.30 & 0.25 & 1.09 & 0.86 & 2.22 & 7.26 \\
3 & 0.62 & 0.47 & 0.30 & 0.25 & 1.63 & 1.18 & 1.80 & 10.14 \\
4 & 0.20 & 0.22 & 0.30 & 0.25 & 0.97 & 0.69 & 2.07 & 19.45 \\
5 & 0.27 & 0.27 & 0.30 & 0.25 & 1.09 & 0.80 & 1.73 & 9.48 \\
6 & 0.33 & 0.41 & 0.30 & 0.25 & 1.30 & 1.08 & 2.68 & 15.14 \\
7 & 0.50 & 0.43 & 0.30 & 0.25 & 1.48 & 1.10 & 2.17 & 14.02 \\
8 & 0.33 & 0.35 & 0.30 & 0.25 & 1.23 & 0.95 & 2.30 & 11.62 \\
9 & 0.33 & 0.32 & 0.30 & 0.25 & 1.20 & 0.88 & 2.26 & 21.74 \\
10 & 0.45 & 0.37 & 0.30 & 0.25 & 1.37 & 0.98 & 2.16 & 7.35 \\
11 & 0.50 & 0.33 & 0.30 & 0.25 & 1.38 & 0.92 & 1.90 & 6.15 \\
12 & 0.07 & 0.26 & 0.30 & 0.25 & 0.88 & 0.77 & 1.98 & 18.80 \\
13 & 0.25 & 0.38 & 0.30 & 0.25 & 1.18 & 1.02 & 2.15 & 13.90 \\
\hline
\end{tabular}

Table 10: Instance PMDd: data.

\begin{tabular}{ccccccc|cc} 
& \multicolumn{2}{c}{ Table 10: Instance PMDd: data. } \\
customer & $T_{i}^{\text {dep }}$ & $T_{i}^{\text {disp }}$ & $T_{i}^{\text {disp,dep }}$ & $T_{i}^{\text {unl }}$ & $T_{i}^{1}$ & $T_{i}^{2}$ & $S_{i}$ & $Q_{i}$ \\
\hline 1 & 0.43 & 0.26 & 0.30 & 0.25 & 1.24 & 0.77 & 1.97 & 11.82 \\
2 & 0.24 & 0.30 & 0.30 & 0.25 & 1.09 & 0.86 & 2.22 & 5.73 \\
3 & 0.62 & 0.47 & 0.30 & 0.25 & 1.63 & 1.18 & 1.80 & 17.07 \\
4 & 0.20 & 0.22 & 0.30 & 0.25 & 0.97 & 0.69 & 2.07 & 14.19 \\
5 & 0.27 & 0.27 & 0.30 & 0.25 & 1.09 & 0.80 & 1.73 & 10.21 \\
6 & 0.33 & 0.41 & 0.30 & 0.25 & 1.30 & 1.08 & 2.68 & 20.12 \\
7 & 0.50 & 0.43 & 0.30 & 0.25 & 1.48 & 1.10 & 2.17 & 2.29 \\
8 & 0.33 & 0.35 & 0.30 & 0.25 & 1.23 & 0.95 & 2.30 & 10.12 \\
9 & 0.33 & 0.32 & 0.30 & 0.25 & 1.20 & 0.88 & 2.26 & 4.51 \\
10 & 0.45 & 0.37 & 0.30 & 0.25 & 1.37 & 0.98 & 2.16 & 14.30 \\
11 & 0.50 & 0.33 & 0.30 & 0.25 & 1.38 & 0.92 & 1.90 & 20.40 \\
12 & 0.07 & 0.26 & 0.30 & 0.25 & 0.88 & 0.77 & 1.98 & 21.54 \\
13 & 0.25 & 0.38 & 0.30 & 0.25 & 1.18 & 1.02 & 2.15 & 0.33 \\
\hline
\end{tabular}


Table 11: Instance PMD.a: data.

\begin{tabular}{ccccccc|cc} 
& \multicolumn{2}{c}{ Table 11: Instance PMD.a: data. } \\
customer & $T_{i}^{\text {dep }}$ & $T_{i}^{\text {disp }}$ & $T_{i}^{\text {disp,dep }}$ & $T_{i}^{\text {unl }}$ & $T_{i}^{1}$ & $T_{i}^{2}$ & $S_{i}$ & $Q_{i}$ \\
\hline 1 & 0.45 & 0.63 & 0.25 & 0.25 & 1.59 & 1.52 & 1.97 & 9.04 \\
2 & 0.54 & 0.49 & 0.25 & 0.25 & 1.53 & 1.23 & 2.22 & 19.23 \\
3 & 0.54 & 0.39 & 0.25 & 0.25 & 1.43 & 1.02 & 1.80 & 21.54 \\
4 & 0.45 & 0.31 & 0.25 & 0.25 & 1.26 & 0.88 & 2.07 & 13.27 \\
5 & 0.64 & 0.48 & 0.25 & 0.25 & 1.62 & 1.20 & 1.73 & 4.54 \\
6 & 0.46 & 0.50 & 0.25 & 0.25 & 1.46 & 1.25 & 2.68 & 6.35 \\
7 & 0.31 & 0.08 & 0.25 & 0.25 & 0.89 & 0.42 & 2.17 & 5.73 \\
8 & 0.44 & 0.47 & 0.25 & 0.25 & 1.41 & 1.19 & 2.30 & 6.38 \\
9 & 0.28 & 0.05 & 0.25 & 0.25 & 0.83 & 0.34 & 2.26 & 4.88 \\
10 & 0.59 & 0.70 & 0.25 & 0.25 & 1.79 & 1.65 & 2.16 & 15.08 \\
11 & 0.44 & 0.45 & 0.25 & 0.25 & 1.39 & 1.14 & 1.90 & 11.38 \\
12 & 0.05 & 0.30 & 0.25 & 0.25 & 0.85 & 0.85 & 1.98 & 5.62 \\
13 & 0.45 & 0.51 & 0.25 & 0.25 & 1.46 & 1.27 & 2.15 & 11.31 \\
\hline
\end{tabular}

Table 12: Instance PMD.b: data.

\begin{tabular}{ccccccc|cc} 
& \multicolumn{7}{c}{ Table 12 Instance PMD.b: data. } \\
customer & $T_{i}^{\text {dep }}$ & $T_{i}^{\text {disp }}$ & $T_{i}^{\text {disp,dep }}$ & $T_{i}^{\text {unl }}$ & $T_{i}^{1}$ & $T_{i}^{2}$ & $S_{i}$ & $Q_{i}$ \\
\hline 1 & 0.27 & 0.41 & 0.17 & 0.25 & 1.09 & 1.06 & 1.97 & 10.96 \\
2 & 0.48 & 0.46 & 0.17 & 0.25 & 1.35 & 1.17 & 2.22 & 8.21 \\
3 & 0.54 & 0.58 & 0.17 & 0.25 & 1.53 & 1.41 & 1.80 & 18.90 \\
4 & 0.64 & 0.56 & 0.17 & 0.25 & 1.61 & 1.36 & 2.07 & 3.30 \\
5 & 0.07 & 0.16 & 0.17 & 0.25 & 0.65 & 0.57 & 1.73 & 2.30 \\
6 & 0.41 & 0.34 & 0.17 & 0.25 & 1.17 & 0.94 & 2.68 & 17.37 \\
7 & 0.39 & 0.48 & 0.17 & 0.25 & 1.29 & 1.20 & 2.17 & 8.40 \\
8 & 0.13 & 0.05 & 0.17 & 0.25 & 0.60 & 0.35 & 2.30 & 3.06 \\
9 & 0.39 & 0.37 & 0.17 & 0.25 & 1.17 & 0.98 & 2.26 & 6.46 \\
10 & 0.41 & 0.47 & 0.17 & 0.25 & 1.30 & 1.20 & 2.16 & 8.12 \\
11 & 0.24 & 0.19 & 0.17 & 0.25 & 0.85 & 0.64 & 1.90 & 19.39 \\
12 & 0.26 & 0.15 & 0.17 & 0.25 & 0.83 & 0.55 & 1.98 & 13.64 \\
13 & 0.50 & 0.37 & 0.17 & 0.25 & 1.28 & 0.99 & 2.15 & 6.48 \\
\hline
\end{tabular}

Table 13: Instance PMD.c: data.

\begin{tabular}{ccccccc|cc} 
& \multicolumn{7}{c}{ Table 13: Instance PMD.c: data. } \\
customer & $T_{i}^{\text {dep }}$ & $T_{i}^{\text {disp }}$ & $T_{i}^{\text {disp }, \text { dep }}$ & $T_{i}^{\text {unl }}$ & $T_{i}^{1}$ & $T_{i}^{2}$ & $S_{i}$ & $Q_{i}$ \\
\hline 1 & 0.29 & 0.31 & 0.20 & 0.25 & 1.05 & 0.88 & 1.97 & 21.28 \\
2 & 0.62 & 0.67 & 0.20 & 0.25 & 1.74 & 1.59 & 2.22 & 7.26 \\
3 & 0.36 & 0.34 & 0.20 & 0.25 & 1.14 & 0.92 & 1.80 & 10.14 \\
4 & 0.47 & 0.53 & 0.20 & 0.25 & 1.44 & 1.30 & 2.07 & 19.45 \\
5 & 0.31 & 0.39 & 0.20 & 0.25 & 1.15 & 1.04 & 1.73 & 9.48 \\
6 & 0.44 & 0.41 & 0.20 & 0.25 & 1.30 & 1.07 & 2.68 & 15.14 \\
7 & 0.14 & 0.27 & 0.20 & 0.25 & 0.86 & 0.79 & 2.17 & 14.02 \\
8 & 0.12 & 0.11 & 0.20 & 0.25 & 0.68 & 0.47 & 2.30 & 11.62 \\
9 & 0.59 & 0.41 & 0.20 & 0.25 & 1.45 & 1.07 & 2.26 & 21.74 \\
10 & 0.07 & 0.22 & 0.20 & 0.25 & 0.74 & 0.69 & 2.16 & 7.35 \\
11 & 0.63 & 0.76 & 0.20 & 0.25 & 1.85 & 1.78 & 1.90 & 6.15 \\
12 & 0.42 & 0.27 & 0.20 & 0.25 & 1.14 & 0.79 & 1.98 & 18.80 \\
13 & 0.33 & 0.52 & 0.20 & 0.25 & 1.30 & 1.29 & 2.15 & 13.90 \\
\hline
\end{tabular}

Table 14: Instance PMD.d: data.

\begin{tabular}{ccccccc|cc} 
& \multicolumn{7}{c}{ Table 14: Instance PMD.d: data. } \\
customer & $T_{i}^{\text {dep }}$ & $T_{i}^{\text {disp }}$ & $T_{i}^{\text {disp,dep }}$ & $T_{i}^{\text {unl }}$ & $T_{i}^{1}$ & $T_{i}^{2}$ & $S_{i}$ & $Q_{i}$ \\
\hline 1 & 0.20 & 0.28 & 0.30 & 0.25 & 1.04 & 0.82 & 1.97 & 11.82 \\
2 & 0.60 & 0.60 & 0.30 & 0.25 & 1.75 & 1.46 & 2.22 & 5.73 \\
3 & 0.51 & 0.33 & 0.30 & 0.25 & 1.39 & 0.90 & 1.80 & 17.07 \\
4 & 0.03 & 0.31 & 0.30 & 0.25 & 0.89 & 0.87 & 2.07 & 14.19 \\
5 & 0.50 & 0.54 & 0.30 & 0.25 & 1.60 & 1.34 & 1.73 & 10.21 \\
6 & 0.62 & 0.61 & 0.30 & 0.25 & 1.79 & 1.48 & 2.68 & 20.12 \\
7 & 0.41 & 0.15 & 0.30 & 0.25 & 1.11 & 0.56 & 2.17 & 2.29 \\
8 & 0.60 & 0.51 & 0.30 & 0.25 & 1.65 & 1.26 & 2.30 & 10.12 \\
9 & 0.03 & 0.29 & 0.30 & 0.25 & 0.86 & 0.83 & 2.26 & 4.51 \\
10 & 0.03 & 0.29 & 0.30 & 0.25 & 0.87 & 0.84 & 2.16 & 14.30 \\
11 & 0.33 & 0.41 & 0.30 & 0.25 & 1.29 & 1.07 & 1.90 & 20.40 \\
12 & 0.28 & 0.35 & 0.30 & 0.25 & 1.18 & 0.95 & 1.98 & 21.54 \\
13 & 0.43 & 0.25 & 0.30 & 0.25 & 1.23 & 0.76 & 2.15 & 0.33 \\
\hline
\end{tabular}

Table 15: Instance PCb: data.

\begin{tabular}{ccccccc|cc} 
& \multicolumn{7}{c}{ Table 15 : Instance PCb: data. } \\
customer & $T_{i}^{\text {dep }}$ & $T_{i}^{\text {disp }}$ & $T_{i}^{\text {disp,dep }}$ & $T_{i}^{\text {unl }}$ & $T_{i}^{1}$ & $T_{i}^{2}$ & $S_{i}$ & $Q_{i}$ \\
\hline 1 & 0.65 & 0.55 & 0.48 & 0.25 & 1.93 & 1.35 & 0.35 & 161.28 \\
2 & 0.97 & 1.05 & 0.48 & 0.25 & 2.75 & 2.35 & 0.52 & 69.68 \\
3 & 0.47 & 0.37 & 0.48 & 0.25 & 1.57 & 0.98 & 0.48 & 113.91 \\
4 & 0.62 & 0.78 & 0.48 & 0.25 & 2.13 & 1.82 & 0.28 & 57.52 \\
5 & 0.43 & 0.42 & 0.48 & 0.25 & 1.58 & 1.08 & 0.48 & 146.30 \\
6 & 0.23 & 0.32 & 0.48 & 0.25 & 1.28 & 0.88 & 0.44 & 138.68 \\
7 & 0.45 & 0.60 & 0.48 & 0.25 & 1.78 & 1.45 & 0.47 & 51.15 \\
8 & 0.60 & 0.67 & 0.48 & 0.25 & 2.00 & 1.58 & 0.39 & 178.64 \\
9 & 0.28 & 0.40 & 0.48 & 0.25 & 1.42 & 1.05 & 0.37 & 189.70 \\
10 & 0.35 & 0.50 & 0.48 & 0.25 & 1.58 & 1.25 & 0.52 & 185.27 \\
11 & 0.45 & 0.48 & 0.48 & 0.25 & 1.67 & 1.22 & 0.65 & 34.25 \\
12 & 0.92 & 1.05 & 0.48 & 0.25 & 2.70 & 2.35 & 0.50 & 69.95 \\
13 & 0.47 & 0.60 & 0.48 & 0.25 & 1.80 & 1.45 & 0.47 & 92.86 \\
14 & 0.28 & 0.53 & 0.48 & 0.25 & 1.55 & 1.32 & 0.52 & 153.04 \\
15 & 0.27 & 0.33 & 0.48 & 0.25 & 1.33 & 0.92 & 0.49 & 179.86 \\
16 & 0.50 & 0.85 & 0.48 & 0.25 & 2.08 & 1.95 & 0.43 & 46.65 \\
17 & 0.27 & 0.22 & 0.48 & 0.25 & 1.22 & 0.68 & 0.45 & 113.16 \\
18 & 0.67 & 0.75 & 0.48 & 0.25 & 2.15 & 1.75 & 0.47 & 136.24 \\
19 & 0.45 & 0.22 & 0.48 & 0.25 & 1.40 & 0.68 & 0.58 & 58.05 \\
20 & 0.48 & 0.53 & 0.48 & 0.25 & 1.75 & 1.32 & 0.38 & 53.52 \\
21 & 0.13 & 0.48 & 0.48 & 0.25 & 1.35 & 1.22 & 0.29 & 43.90 \\
22 & 0.60 & 0.60 & 0.48 & 0.25 & 1.93 & 1.45 & 0.41 & 76.92 \\
23 & 0.73 & 1.05 & 0.48 & 0.25 & 2.52 & 2.35 & 0.51 & 150.57 \\
24 & 0.48 & 0.43 & 0.48 & 0.25 & 1.65 & 1.12 & 0.54 & 124.50 \\
25 & 0.77 & 0.90 & 0.48 & 0.25 & 2.40 & 2.05 & 0.40 & 166.73 \\
26 & 0.55 & 0.78 & 0.48 & 0.25 & 2.07 & 1.82 & 0.34 & 179.86 \\
27 & 0.42 & 0.65 & 0.48 & 0.25 & 1.80 & 1.55 & 0.37 & 174.82 \\
28 & 0.52 & 0.72 & 0.48 & 0.25 & 1.97 & 1.68 & 0.37 & 105.76 \\
29 & 0.22 & 0.37 & 0.48 & 0.25 & 1.32 & 0.98 & 0.44 & 32.69 \\
\hline & & & & & & & &
\end{tabular}


Table 16: Instance PCc: data.

\begin{tabular}{ccccccc|cc}
\multicolumn{7}{c}{ Table 16: Instance PCc: data. } \\
customer & $T_{i}^{\text {dep }}$ & $T_{i}^{\text {disp }}$ & $T_{i}^{\text {disp,dep }}$ & $T_{i}^{\text {unl }}$ & $T_{i}^{1}$ & $T_{i}^{2}$ & $S_{i}$ & $Q_{i}$ \\
\hline 1 & 0.65 & 0.55 & 0.48 & 0.25 & 1.93 & 1.35 & 0.35 & 22.24 \\
2 & 0.97 & 1.05 & 0.48 & 0.25 & 2.75 & 2.35 & 0.52 & 152.27 \\
3 & 0.47 & 0.37 & 0.48 & 0.25 & 1.57 & 0.98 & 0.48 & 134.61 \\
4 & 0.62 & 0.78 & 0.48 & 0.25 & 2.13 & 1.82 & 0.28 & 86.95 \\
5 & 0.43 & 0.42 & 0.48 & 0.25 & 1.58 & 1.08 & 0.48 & 58.43 \\
6 & 0.23 & 0.32 & 0.48 & 0.25 & 1.28 & 0.88 & 0.44 & 129.81 \\
7 & 0.45 & 0.60 & 0.48 & 0.25 & 1.78 & 1.45 & 0.47 & 165.75 \\
8 & 0.60 & 0.67 & 0.48 & 0.25 & 2.00 & 1.58 & 0.39 & 44.86 \\
9 & 0.28 & 0.40 & 0.48 & 0.25 & 1.42 & 1.05 & 0.37 & 120.58 \\
10 & 0.35 & 0.50 & 0.48 & 0.25 & 1.58 & 1.25 & 0.52 & 114.98 \\
11 & 0.45 & 0.48 & 0.48 & 0.25 & 1.67 & 1.22 & 0.65 & 49.64 \\
12 & 0.92 & 1.05 & 0.48 & 0.25 & 2.70 & 2.35 & 0.50 & 163.95 \\
13 & 0.47 & 0.60 & 0.48 & 0.25 & 1.80 & 1.45 & 0.47 & 33.61 \\
14 & 0.28 & 0.53 & 0.48 & 0.25 & 1.55 & 1.32 & 0.52 & 123.57 \\
15 & 0.27 & 0.33 & 0.48 & 0.25 & 1.33 & 0.92 & 0.49 & 127.08 \\
16 & 0.50 & 0.85 & 0.48 & 0.25 & 2.08 & 1.95 & 0.43 & 156.95 \\
17 & 0.27 & 0.22 & 0.48 & 0.25 & 1.22 & 0.68 & 0.45 & 97.96 \\
18 & 0.67 & 0.75 & 0.48 & 0.25 & 2.15 & 1.75 & 0.47 & 74.46 \\
19 & 0.45 & 0.22 & 0.48 & 0.25 & 1.40 & 0.68 & 0.58 & 33.64 \\
20 & 0.48 & 0.53 & 0.48 & 0.25 & 1.75 & 1.32 & 0.38 & 50.16 \\
21 & 0.13 & 0.48 & 0.48 & 0.25 & 1.35 & 1.22 & 0.29 & 95.24 \\
22 & 0.60 & 0.60 & 0.48 & 0.25 & 1.93 & 1.45 & 0.41 & 36.73 \\
23 & 0.73 & 1.05 & 0.48 & 0.25 & 2.52 & 2.35 & 0.51 & 36.53 \\
24 & 0.48 & 0.43 & 0.48 & 0.25 & 1.65 & 1.12 & 0.54 & 144.58 \\
25 & 0.77 & 0.90 & 0.48 & 0.25 & 2.40 & 2.05 & 0.40 & 156.40 \\
26 & 0.55 & 0.78 & 0.48 & 0.25 & 2.07 & 1.82 & 0.34 & 83.29 \\
27 & 0.42 & 0.65 & 0.48 & 0.25 & 1.80 & 1.55 & 0.37 & 114.22 \\
28 & 0.52 & 0.72 & 0.48 & 0.25 & 1.97 & 1.68 & 0.37 & 155.14 \\
29 & 0.22 & 0.37 & 0.48 & 0.25 & 1.32 & 0.98 & 0.44 & 85.84 \\
\hline
\end{tabular}

Table 17: Instance PCd: data.

\begin{tabular}{ccccccc|cc} 
& \multicolumn{7}{c}{ Table 17: Instance PCd: data. } \\
customer & $T_{i}^{\text {dep }}$ & $T_{i}^{\text {disp }}$ & $T_{i}^{\text {disp }, \text { dep }}$ & $T_{i}^{\text {unl }}$ & $T_{i}^{1}$ & $T_{i}^{2}$ & $S_{i}$ & $Q_{i}$ \\
\hline 1 & 0.65 & 0.55 & 0.48 & 0.25 & 1.93 & 1.35 & 0.35 & 22.98 \\
2 & 0.97 & 1.05 & 0.48 & 0.25 & 2.75 & 2.35 & 0.52 & 70.40 \\
3 & 0.47 & 0.37 & 0.48 & 0.25 & 1.57 & 0.98 & 0.48 & 11.06 \\
4 & 0.62 & 0.78 & 0.48 & 0.25 & 2.13 & 1.82 & 0.28 & 158.58 \\
5 & 0.43 & 0.42 & 0.48 & 0.25 & 1.58 & 1.08 & 0.48 & 119.06 \\
6 & 0.23 & 0.32 & 0.48 & 0.25 & 1.28 & 0.88 & 0.44 & 33.85 \\
7 & 0.45 & 0.60 & 0.48 & 0.25 & 1.78 & 1.45 & 0.47 & 107.36 \\
8 & 0.60 & 0.67 & 0.48 & 0.25 & 2.00 & 1.58 & 0.39 & 134.50 \\
9 & 0.28 & 0.40 & 0.48 & 0.25 & 1.42 & 1.05 & 0.37 & 77.68 \\
10 & 0.35 & 0.50 & 0.48 & 0.25 & 1.58 & 1.25 & 0.52 & 28.19 \\
11 & 0.45 & 0.48 & 0.48 & 0.25 & 1.67 & 1.22 & 0.65 & 154.24 \\
12 & 0.92 & 1.05 & 0.48 & 0.25 & 2.70 & 2.35 & 0.50 & 16.32 \\
13 & 0.47 & 0.60 & 0.48 & 0.25 & 1.80 & 1.45 & 0.47 & 133.37 \\
14 & 0.28 & 0.53 & 0.48 & 0.25 & 1.55 & 1.32 & 0.52 & 29.73 \\
15 & 0.27 & 0.33 & 0.48 & 0.25 & 1.33 & 0.92 & 0.49 & 156.24 \\
16 & 0.50 & 0.85 & 0.48 & 0.25 & 2.08 & 1.95 & 0.43 & 52.52 \\
17 & 0.27 & 0.22 & 0.48 & 0.25 & 1.22 & 0.68 & 0.45 & 105.25 \\
18 & 0.67 & 0.75 & 0.48 & 0.25 & 2.15 & 1.75 & 0.47 & 115.45 \\
19 & 0.45 & 0.22 & 0.48 & 0.25 & 1.40 & 0.68 & 0.58 & 14.68 \\
20 & 0.48 & 0.53 & 0.48 & 0.25 & 1.75 & 1.32 & 0.38 & 109.37 \\
21 & 0.13 & 0.48 & 0.48 & 0.25 & 1.35 & 1.22 & 0.29 & 136.36 \\
22 & 0.60 & 0.60 & 0.48 & 0.25 & 1.93 & 1.45 & 0.41 & 64.28 \\
23 & 0.73 & 1.05 & 0.48 & 0.25 & 2.52 & 2.35 & 0.51 & 26.02 \\
24 & 0.48 & 0.43 & 0.48 & 0.25 & 1.65 & 1.12 & 0.54 & 102.81 \\
25 & 0.77 & 0.90 & 0.48 & 0.25 & 2.40 & 2.05 & 0.40 & 16.59 \\
26 & 0.55 & 0.78 & 0.48 & 0.25 & 2.07 & 1.82 & 0.34 & 48.20 \\
27 & 0.42 & 0.65 & 0.48 & 0.25 & 1.80 & 1.55 & 0.37 & 144.03 \\
28 & 0.52 & 0.72 & 0.48 & 0.25 & 1.97 & 1.68 & 0.37 & 92.28 \\
29 & 0.22 & 0.37 & 0.48 & 0.25 & 1.32 & 0.98 & 0.44 & 36.33 \\
\hline
\end{tabular}

\begin{tabular}{ccccccc|cc} 
& \multicolumn{7}{c}{ Table 18: Instance PC.a: data. } \\
customer & $T_{i}^{\text {dep }}$ & $T_{i}^{\text {disp }}$ & $T_{i}^{\text {disp,dep }}$ & $T_{i}^{\text {unl }}$ & $T_{i}^{1}$ & $T_{i}^{2}$ & $S_{i}$ & $Q_{i}$ \\
\hline 1 & 0.46 & 0.58 & 0.30 & 0.25 & 1.59 & 1.41 & 0.35 & 44.17 \\
2 & 0.48 & 0.73 & 0.30 & 0.25 & 1.76 & 1.71 & 0.52 & 13.75 \\
3 & 0.38 & 0.26 & 0.30 & 0.25 & 1.19 & 0.78 & 0.48 & 67.92 \\
4 & 0.07 & 0.24 & 0.30 & 0.25 & 0.86 & 0.73 & 0.28 & 98.33 \\
5 & 0.48 & 0.19 & 0.30 & 0.25 & 1.22 & 0.63 & 0.48 & 42.50 \\
6 & 0.13 & 0.18 & 0.30 & 0.25 & 0.86 & 0.61 & 0.44 & 214.17 \\
7 & 0.36 & 0.19 & 0.30 & 0.25 & 1.10 & 0.64 & 0.47 & 45.83 \\
8 & 0.61 & 0.31 & 0.30 & 0.25 & 1.47 & 0.87 & 0.39 & 198.33 \\
9 & 0.77 & 0.79 & 0.30 & 0.25 & 2.12 & 1.84 & 0.37 & 152.50 \\
10 & 0.21 & 0.31 & 0.30 & 0.25 & 1.08 & 0.88 & 0.52 & 31.33 \\
11 & 0.62 & 0.60 & 0.30 & 0.25 & 1.78 & 1.46 & 0.65 & 59.83 \\
12 & 0.35 & 0.62 & 0.30 & 0.25 & 1.52 & 1.49 & 0.50 & 100.83 \\
13 & 0.10 & 0.22 & 0.30 & 0.25 & 0.87 & 0.68 & 0.47 & 49.17 \\
14 & 0.97 & 0.77 & 0.30 & 0.25 & 2.29 & 1.79 & 0.52 & 75.00 \\
15 & 0.65 & 0.47 & 0.30 & 0.25 & 1.67 & 1.19 & 0.49 & 64.67 \\
16 & 0.75 & 0.61 & 0.30 & 0.25 & 1.91 & 1.46 & 0.43 & 89.17 \\
17 & 0.35 & 0.47 & 0.30 & 0.25 & 1.37 & 1.19 & 0.45 & 53.33 \\
18 & 0.42 & 0.52 & 0.30 & 0.25 & 1.49 & 1.29 & 0.47 & 48.58 \\
19 & 0.69 & 0.79 & 0.30 & 0.25 & 2.04 & 1.84 & 0.58 & 122.00 \\
20 & 0.97 & 0.68 & 0.30 & 0.25 & 2.20 & 1.60 & 0.38 & 117.92 \\
21 & 0.50 & 0.52 & 0.30 & 0.25 & 1.57 & 1.29 & 0.29 & 62.50 \\
22 & 0.05 & 0.32 & 0.30 & 0.25 & 0.92 & 0.89 & 0.41 & 69.17 \\
23 & 0.99 & 0.73 & 0.30 & 0.25 & 2.28 & 1.72 & 0.51 & 68.83 \\
24 & 0.24 & 0.09 & 0.30 & 0.25 & 0.88 & 0.43 & 0.54 & 40.00 \\
25 & 0.84 & 0.76 & 0.30 & 0.25 & 2.15 & 1.77 & 0.40 & 44.17 \\
26 & 0.13 & 0.34 & 0.30 & 0.25 & 1.02 & 0.92 & 0.34 & 216.67 \\
27 & 0.35 & 0.48 & 0.30 & 0.25 & 1.38 & 1.21 & 0.37 & 27.92 \\
28 & 0.36 & 0.40 & 0.30 & 0.25 & 1.31 & 1.04 & 0.37 & 69.17 \\
29 & 0.82 & 0.98 & 0.30 & 0.25 & 2.35 & 2.21 & 0.44 & 117.50 \\
\hline
\end{tabular}


Table 19: Instance PC.b: data.

\begin{tabular}{ccccccc|cc} 
& \multicolumn{7}{c}{ Table 19 instance PC.b: data. } \\
customer & $T_{i}^{\text {dep }}$ & $T_{i}^{\text {disp }}$ & $T_{i}^{\text {disp,dep }}$ & $T_{i}^{\text {unl }}$ & $T_{i}^{1}$ & $T_{i}^{2}$ & $S_{i}$ & $Q_{i}$ \\
\hline 1 & 0.80 & 0.99 & 0.25 & 0.25 & 2.29 & 2.22 & 0.35 & 44.17 \\
2 & 0.27 & 0.06 & 0.25 & 0.25 & 0.83 & 0.37 & 0.52 & 13.75 \\
3 & 0.66 & 0.89 & 0.25 & 0.25 & 2.05 & 2.03 & 0.48 & 67.92 \\
4 & 0.79 & 0.96 & 0.25 & 0.25 & 2.25 & 2.18 & 0.28 & 98.33 \\
5 & 0.04 & 0.22 & 0.25 & 0.25 & 0.76 & 0.69 & 0.48 & 42.50 \\
6 & 0.06 & 0.26 & 0.25 & 0.25 & 0.81 & 0.77 & 0.44 & 214.17 \\
7 & 0.20 & 0.18 & 0.25 & 0.25 & 0.87 & 0.61 & 0.47 & 45.83 \\
8 & 0.75 & 0.94 & 0.25 & 0.25 & 2.19 & 2.12 & 0.39 & 198.33 \\
9 & 0.80 & 0.83 & 0.25 & 0.25 & 2.13 & 1.90 & 0.37 & 152.50 \\
10 & 0.51 & 0.67 & 0.25 & 0.25 & 1.67 & 1.59 & 0.52 & 31.33 \\
11 & 0.68 & 0.80 & 0.25 & 0.25 & 1.99 & 1.86 & 0.65 & 59.83 \\
12 & 0.78 & 0.89 & 0.25 & 0.25 & 2.17 & 2.03 & 0.50 & 100.83 \\
13 & 0.71 & 0.57 & 0.25 & 0.25 & 1.78 & 1.39 & 0.47 & 49.17 \\
14 & 0.49 & 0.55 & 0.25 & 0.25 & 1.54 & 1.35 & 0.52 & 75.00 \\
15 & 0.93 & 1.07 & 0.25 & 0.25 & 2.50 & 2.39 & 0.49 & 64.67 \\
16 & 0.18 & 0.33 & 0.25 & 0.25 & 1.01 & 0.90 & 0.43 & 89.17 \\
17 & 0.62 & 0.69 & 0.25 & 0.25 & 1.81 & 1.62 & 0.45 & 53.33 \\
18 & 0.87 & 0.96 & 0.25 & 0.25 & 2.33 & 2.16 & 0.47 & 48.58 \\
19 & 0.67 & 0.47 & 0.25 & 0.25 & 1.63 & 1.18 & 0.58 & 122.00 \\
20 & 0.94 & 0.84 & 0.25 & 0.25 & 2.28 & 1.93 & 0.38 & 117.92 \\
21 & 0.80 & 0.66 & 0.25 & 0.25 & 1.95 & 1.56 & 0.29 & 62.50 \\
22 & 0.53 & 0.63 & 0.25 & 0.25 & 1.66 & 1.51 & 0.41 & 69.17 \\
23 & 0.23 & 0.20 & 0.25 & 0.25 & 0.93 & 0.65 & 0.51 & 68.83 \\
24 & 0.33 & 0.09 & 0.25 & 0.25 & 0.92 & 0.44 & 0.54 & 40.00 \\
25 & 0.83 & 1.06 & 0.25 & 0.25 & 2.39 & 2.37 & 0.40 & 44.17 \\
26 & 0.40 & 0.64 & 0.25 & 0.25 & 1.54 & 1.52 & 0.34 & 216.67 \\
27 & 0.93 & 0.69 & 0.25 & 0.25 & 2.11 & 1.63 & 0.37 & 27.92 \\
28 & 0.59 & 0.69 & 0.25 & 0.25 & 1.78 & 1.62 & 0.37 & 69.17 \\
29 & 0.10 & 0.31 & 0.25 & 0.25 & 0.91 & 0.87 & 0.44 & 117.50 \\
\hline & & & & & & & &
\end{tabular}

Table 20: Instance PC.c: data.

\begin{tabular}{ccccccc|cc} 
& \multicolumn{7}{c}{ Table 20: Instance PC.c: data. } \\
customer & $T_{i}^{\text {dep }}$ & $T_{i}^{\text {disp }}$ & $T_{i}^{\text {disp } \text { dep }}$ & $T_{i}^{\text {unl }}$ & $T_{i}^{1}$ & $T_{i}^{2}$ & $S_{i}$ & $Q_{i}$ \\
\hline 1 & 0.52 & 0.53 & 0.50 & 0.25 & 1.81 & 1.32 & 0.35 & 44.17 \\
2 & 0.23 & 0.41 & 0.50 & 0.25 & 1.39 & 1.06 & 0.52 & 13.75 \\
3 & 0.08 & 0.45 & 0.50 & 0.25 & 1.29 & 1.15 & 0.48 & 67.92 \\
4 & 0.79 & 1.06 & 0.50 & 0.25 & 2.60 & 2.36 & 0.28 & 98.33 \\
5 & 0.58 & 0.08 & 0.50 & 0.25 & 1.41 & 0.41 & 0.48 & 42.50 \\
6 & 0.97 & 0.81 & 0.50 & 0.25 & 2.52 & 1.86 & 0.44 & 214.17 \\
7 & 0.83 & 1.19 & 0.50 & 0.25 & 2.78 & 2.64 & 0.47 & 45.83 \\
8 & 0.98 & 0.97 & 0.50 & 0.25 & 2.71 & 2.20 & 0.39 & 198.33 \\
9 & 0.88 & 1.11 & 0.50 & 0.25 & 2.75 & 2.48 & 0.37 & 152.50 \\
10 & 0.56 & 0.85 & 0.50 & 0.25 & 2.17 & 1.96 & 0.52 & 31.33 \\
11 & 0.88 & 0.44 & 0.50 & 0.25 & 2.07 & 1.14 & 0.65 & 59.83 \\
12 & 0.82 & 0.61 & 0.50 & 0.25 & 2.19 & 1.48 & 0.50 & 100.83 \\
13 & 0.90 & 0.86 & 0.50 & 0.25 & 2.52 & 1.98 & 0.47 & 49.17 \\
14 & 0.61 & 0.57 & 0.50 & 0.25 & 1.93 & 1.38 & 0.52 & 75.00 \\
15 & 0.46 & 0.67 & 0.50 & 0.25 & 1.89 & 1.59 & 0.49 & 64.67 \\
16 & 0.44 & 0.89 & 0.50 & 0.25 & 2.08 & 2.04 & 0.43 & 89.17 \\
17 & 0.85 & 0.44 & 0.50 & 0.25 & 2.04 & 1.14 & 0.45 & 53.33 \\
18 & 0.83 & 1.01 & 0.50 & 0.25 & 2.58 & 2.26 & 0.47 & 48.58 \\
19 & 0.24 & 0.72 & 0.50 & 0.25 & 1.71 & 1.69 & 0.58 & 122.00 \\
20 & 0.20 & 0.31 & 0.50 & 0.25 & 1.26 & 0.87 & 0.38 & 117.92 \\
21 & 0.69 & 1.03 & 0.50 & 0.25 & 2.48 & 2.31 & 0.29 & 62.50 \\
22 & 0.12 & 0.41 & 0.50 & 0.25 & 1.27 & 1.06 & 0.41 & 69.17 \\
23 & 0.08 & 0.48 & 0.50 & 0.25 & 1.31 & 1.21 & 0.51 & 68.83 \\
24 & 0.68 & 0.19 & 0.50 & 0.25 & 1.62 & 0.63 & 0.54 & 40.00 \\
25 & 0.38 & 0.36 & 0.50 & 0.25 & 1.49 & 0.96 & 0.40 & 44.17 \\
26 & 0.85 & 0.44 & 0.50 & 0.25 & 2.04 & 1.13 & 0.34 & 216.67 \\
27 & 0.29 & 0.77 & 0.50 & 0.25 & 1.81 & 1.79 & 0.37 & 27.92 \\
28 & 0.78 & 0.65 & 0.50 & 0.25 & 2.17 & 1.55 & 0.37 & 69.17 \\
29 & 0.15 & 0.39 & 0.50 & 0.25 & 1.29 & 1.03 & 0.44 & 117.50 \\
\hline
\end{tabular}

Table 21: Instance PC.d: data.

\begin{tabular}{ccccccc|cc} 
& \multicolumn{7}{c}{ Table 21: Instance PC.d: data. } \\
customer & $T_{i}^{\text {dep }}$ & $T_{i}^{\text {disp }}$ & $T_{i}^{\text {disp }}$ dep & $T_{i}^{\text {unl }}$ & $T_{i}^{1}$ & $T_{i}^{2}$ & $S_{i}$ & $Q_{i}$ \\
\hline 1 & 0.94 & 0.83 & 1.00 & 0.25 & 3.01 & 1.90 & 0.35 & 44.17 \\
2 & 0.93 & 0.48 & 1.00 & 0.25 & 2.66 & 1.21 & 0.52 & 13.75 \\
3 & 0.42 & 1.38 & 1.00 & 0.25 & 3.05 & 3.00 & 0.48 & 67.92 \\
4 & 0.43 & 1.06 & 1.00 & 0.25 & 2.74 & 2.38 & 0.28 & 98.33 \\
5 & 0.96 & 0.61 & 1.00 & 0.25 & 2.82 & 1.47 & 0.48 & 42.50 \\
6 & 0.83 & 1.67 & 1.00 & 0.25 & 3.76 & 3.60 & 0.44 & 214.17 \\
7 & 0.07 & 1.06 & 1.00 & 0.25 & 2.38 & 2.36 & 0.47 & 45.83 \\
8 & 0.18 & 1.13 & 1.00 & 0.25 & 2.56 & 2.52 & 0.39 & 198.33 \\
9 & 0.32 & 1.16 & 1.00 & 0.25 & 2.73 & 2.57 & 0.37 & 152.50 \\
10 & 0.14 & 1.10 & 1.00 & 0.25 & 2.48 & 2.44 & 0.52 & 31.33 \\
11 & 0.38 & 0.76 & 1.00 & 0.25 & 2.39 & 1.77 & 0.65 & 59.83 \\
12 & 0.96 & 1.52 & 1.00 & 0.25 & 3.73 & 3.29 & 0.50 & 100.83 \\
13 & 0.78 & 0.34 & 1.00 & 0.25 & 2.36 & 0.92 & 0.47 & 49.17 \\
14 & 0.83 & 0.34 & 1.00 & 0.25 & 2.42 & 0.93 & 0.52 & 75.00 \\
15 & 0.10 & 1.06 & 1.00 & 0.25 & 2.41 & 2.37 & 0.49 & 64.67 \\
16 & 0.71 & 1.69 & 1.00 & 0.25 & 3.65 & 3.62 & 0.43 & 89.17 \\
17 & 0.49 & 1.40 & 1.00 & 0.25 & 3.14 & 3.05 & 0.45 & 53.33 \\
18 & 0.27 & 0.88 & 1.00 & 0.25 & 2.39 & 2.00 & 0.47 & 48.58 \\
19 & 0.06 & 1.01 & 1.00 & 0.25 & 2.32 & 2.28 & 0.58 & 122.00 \\
20 & 0.09 & 1.02 & 1.00 & 0.25 & 2.36 & 2.29 & 0.38 & 117.92 \\
21 & 0.16 & 0.90 & 1.00 & 0.25 & 2.31 & 2.05 & 0.29 & 62.50 \\
22 & 0.28 & 1.18 & 1.00 & 0.25 & 2.71 & 2.62 & 0.41 & 69.17 \\
23 & 0.20 & 1.10 & 1.00 & 0.25 & 2.55 & 2.45 & 0.51 & 68.83 \\
24 & 0.98 & 0.33 & 1.00 & 0.25 & 2.55 & 0.91 & 0.54 & 40.00 \\
25 & 0.13 & 0.93 & 1.00 & 0.25 & 2.31 & 2.10 & 0.40 & 44.17 \\
26 & 0.14 & 1.13 & 1.00 & 0.25 & 2.52 & 2.51 & 0.34 & 216.67 \\
27 & 0.42 & 0.60 & 1.00 & 0.25 & 2.27 & 1.45 & 0.37 & 27.92 \\
28 & 0.38 & 1.12 & 1.00 & 0.25 & 2.75 & 2.50 & 0.37 & 69.17 \\
29 & 0.91 & 0.33 & 1.00 & 0.25 & 2.49 & 0.91 & 0.44 & 117.50 \\
\hline
\end{tabular}

